\title{
LA LEGAL ORIGINS THEORY : DROIT, ÉCONOMIE, IDÉOLOGIE
}

\author{
Antoine VANDENBULKE ${ }^{1}$
}

Résumé : Selon la Legal Origins Theory, les origines juridiques d'un État ont une influence significative sur sa croissance économique. Née dans l'orbite du droit des sociétés, elle a d'abord été défendue par un collectif d'auteurs à l'occasion de l'étude du niveau de protection accordé aux actionnaires minoritaires dans différents États à travers le monde. Ils en ont tiré la conclusion que les États appartenant à la culture juridique de Common Law accordaient le plus haut degré de protection, source d'un marché financier efficace et synonyme de croissance économique. La Legal Origins Theory s'est ensuite étendue à d'autres domaines juridiques et s'est attachée à démontrer plus globalement l'efficience générale du modèle de Common Law. Dans la foulée, elle fut au coeur des rapports Doing Business de la Banque mondiale. Cette théorie a suscité de nombreuses critiques que la présente contribution a pour ambition d'exposer. Après l'analyse de la théorie et des critiques émises à son encontre, l'étude tente de dégager les présupposés philosophiques sous-jacents à la Legal Origins Theory, à travers la pensée de deux auteurs : Friedrich von Hayek et Bruno Leoni.

1 Introduction

2 Le point de départ du raisonnement

2.1 Law matters

2.2 Common Law vs. Civil Law

3 La Legal Origins Theory

3.1 Son lieu de naissance : le droit des sociétés

3.1.1 Remise en cause d'un régime universel de l'actionnariat dispersé

3.1.2 Influence des origines juridiques d'un État sur la protection des investisseurs

1. Assistant à l'Université de Liège, Unité de Droit économique et Théorie du droit. Courriel : antoine. vandenbulke@ulg.ac.be. Adresse : Quartier Agora, Place des Orateurs 3 (Bât. B31), 4000 Liège, Belgique. 
3.1.2.1 Droits des actionnaires

3.1.2.2 Droits des créanciers

3.1.2.3 Effectivité de la protection

3.1.2.4 Concentration de la propriété

3.1.3 Influence des origines juridiques sur le développement des marchés financiers

3.2 Sa généralisation : un système globalement efficient

3.3 Son impact politique : les rapports Doing Business de la Banque mondiale

4 Les critiques émises à l'encontre de la Legal Origins Theory

4.1 Une méthode faussement objective

4.2 Une extrapolation hasardeuse à partir du droit comparé

4.3 Un modèle universellement pertinent?

4.4 Does law really matter?

4.5 Les qualités intrinsèques du modèle de Civil Law

4.6 Le droit n'a pas à être inféodé à l'économie

5 Les présupposés philosophiques de la Legal Origins Theory: la place de l'idéologie dans la pensée économique et juridique ou l'impossible objectivité

5.1 Friedrich August von Hayek

5.2 Bruno Leoni

6 Conclusion

\section{INTRODUCTION}

La Legal Origins Theory soutient que les origines juridiques d'un État - principalement dues à son héritage colonial - seraient un facteur déterminant de sa croissance économique. Initialement développée par des économistes américains dans les années 1990, cette théorie a tout d'abord été appliquée au droit des sociétés et plus particulièrement au degré de protection accordé aux actionnaires minoritaires. Ces chercheurs en ont tiré la conclusion que les États appartenant à la culture juridique de Common Law accordaient le plus haut degré de protection, source d'un marché financier efficace et synonyme de croissance économique. Dans les années 2000, la théorie s'est étendue à d'autres domaines juridiques et s'est attachée à démontrer plus globalement l'efficience générale du modèle de Common Law. Dans la foulée, cette théorie a fortement influencé les très commentés rapports Doing Business de la Banque mondiale, dont l'objectif éminemment politique est la mise sur pied d'un cadre juridique favorable aux attentes du secteur privé.

Ces différents rapports et études empiriques ont entraîné de vives critiques de la part des experts du monde juridique et économique. Si cette théorie a fait couler beaucoup d'encre en littérature anglophone, elle a pourtant été jusqu'ici peu traitée en langue française, malgré les nombreuses réactions qu'elle a suscitées parmi les adeptes du modèle de Civil Law. La présente contribution a donc pour objet de 
présenter cette théorie, d'en exposer les critiques et d'en débusquer les présupposés idéologiques sous-jacents.

Dans un premier temps, nous poserons les jalons nécessaires à la bonne compréhension de ce discours en le situant dans le contexte d'où il est issu : celui de l'analyse économique du droit - Law and Economics - et du droit comparé. La Legal Origins Theory se fonde en effet sur des postulats qu'il est nécessaire d'exposer préalablement (2.).

Nous présenterons ensuite la théorie en tant que telle : les premières études en droit des sociétés qui l'ont fait éclore, sa généralisation à d'autres domaines juridiques et l'impact politique dont elle a pu jouir grâce à son influence sur les rapports Doing Business de la Banque mondiale (3.).

Nous exposerons ensuite les nombreuses critiques émises à son encontre. Celles-ci portent sur la méthodologie adoptée, dénoncent la méconnaissance dont les méthodes du droit comparé ont fait l'objet, soulignent l'impossibilité de définir un modèle universellement pertinent, relativisent l'impact du droit sur l'économie et enfin refusent l'idée même d'une nécessaire efficience du droit, bien que certains auteurs se soient également attelés à soutenir l'efficience du Civil Law, en exposant ses qualités intrinsèques (4.).

Nous aborderons enfin les présupposés de la Legal Origins Theory, c'est-à-dire le cadre idéologique dans lequel cette théorie fut élaborée. Nous nous concentrerons en particulier sur deux auteurs : Bruno Leoni et Friedrich August von Hayek. Ce sera l'occasion de montrer que les débats autour de la Legal Origins Theory sont essentiellement conditionnés par des choix de valeurs et non par un quelconque désaccord scientifique sur les faits ou les données de l'expérience (5).

Nous conclurons dès lors logiquement par une réflexion sur la notion d'objectivité dans ces matières et la difficulté, voire l'impossibilité, de développer une théorie axiologiquement neutre, en tout cas dans le domaine des sciences sociales. Ce faisant, il ne s'agit certes pas de discréditer la théorie, mais d'en mesurer l'exacte portée en la référant au système de valeurs dont elle est, consciemment ou non, issue (6).

\section{LE POINT DE DÉPART DU RAISONNEMENT}

La Legal Origins Theory mobilise principalement deux disciplines : l'analyse économique du droit et le droit comparé ${ }^{2}$. Elle part en effet du présupposé que le droit affecte l'économie (2.1.) et se réfère aux théories de grands comparatistes pour classifier les États en familles (ou cultures) juridiques (2.2.).

2. La combinaison de ces deux disciplines a parfois même pu être considérée comme une discipline à part entière : la Comparative Law and Economics (G. DE GEEST (ed.), Economics of Comparative Law, Cheltenham, Edward Elgar Publishing, 2009). 


\subsection{Law matters}

La Legal Origins Theory se fonde sur une première hypothèse soutenue dans la plupart des cénacles de l'analyse économique du droit: les règles et institutions juridiques d'un État ont une influence sur sa croissance économique ; en d'autres termes, Law matters. Dans sa version néo-classique, la Law and Economics ${ }^{3}$ propose en effet de mesurer l'efficience économique du droit.

Traditionnellement, pourtant, l'économie se définissait en tant que matière et non en tant que méthode. Quant au droit, si ses effets sur l'économie étaient reconnus, il n'était toutefois pas pensé comme un système économique en soi, c'est-àdire « in the sense of a site of activity that could profitably be studied in economic terms $»^{4}$. Dans les années 1960, les premiers travaux de R. Coase ${ }^{5}$ sur la question du social cost et ceux de G. Calabresi ${ }^{6}$ à propos des torts - qui marquèrent l'éclosion de l'analyse économique du droit moderne - vinrent bouleverser la donne. Alors qu'auparavant la science économique était très peu utilisée dans l'étude du droit (à l'exception du droit antitrust ${ }^{7}$ ), elle prétendait désormais servir d'étalon pour mesurer l'efficacité des normes juridiques, dans des secteurs de plus en plus variés. Le droit est dès lors considéré comme doté d'une influence primordiale sur l'économie 8 .

Mesurer l'impact des règles juridiques sur l'économie n'en est pas moins une tâche particulièrement hardie. En effet, une société économiquement prospère l'est-elle grâce à ses institutions juridiques ou, à l'inverse, un État s'est-il doté d'institutions juridiques performantes car il dispose des moyens nécessaires pour les mettre en place et les faire fonctionner - à moins, bien entendu, que la cause principale ne se trouve dans un troisième facteur? L'interconnexion entre droit et société peut difficilement être démêlée. L'ingénieuse idée des tenants de la Legal Origins Theory fut d'observer le droit non pas comme un phénomène endogène à

3. Pour reprendre l'expression anglo-saxonne d'où est issue cette discipline.

4. R. Posner, Economic Analysis of Law, 8th ed., New York, Aspen Publishers, 2011, p. 30. Pour illustrer cette vision du droit, R. Posner la compare à celle du climat : le climat affecte bel et bien l'économie, mais cela n'en fait cependant pas un système économique en soi (idem).

5. R.H. Coase, «The Problem of Social Cost », Journal of Laws and Economics, vol. 3, 1960, pp. 1 et $\mathrm{s}$.

6. G. CAlabresi, « Some Thoughts on Risk Distribution and the Law of Torts », Yale Law Journal, vol. $70, \mathrm{n}^{\circ} 4,1961$, pp. 491-553.

7. Soulignons également que, dès le XVIII siècle, Jérémy Bentham (not. J. Bentham, An Introduction to the Principles of Moral and Legislation, London, T. Payne, 1789 ; J. Bentham, Panopticon ; or, the Inspection House [...] a series of letters, written in the year 1787, London, T. Payne, 1791) et Cesare Beccaria (C. BeCCARIA, Dei delitti e delle pene, Livorno, Marco Coltellini, 1764) étudièrent d'un point de vue économique des questions de droit pénal.

8. Cette idée n'est pas totalement étrangère à la littérature de droit comparé, plus particulièrement à son versant fonctionnel (soutenu notamment par K. Zweigert et H. Kötz - cf. infra point 2.2. - dont la pensée a influencé le mouvement de la Legal Origins Theory) qui appréhende le droit comme une réponse à un problème social. Plus précisément, la méthode comparée fonctionnelle consiste à analyser la manière dont un problème factuel identique est traité juridiquement par les différents systèmes étudiés. 
une société donnée, mais comme un élément extérieur - exogène - aux sociétés qu'il régit ${ }^{9}$. Hormis les grandes puissances colonisatrices (c'est-à-dire l'Angleterre et la France ainsi que, dans une certaine mesure, l'Allemagne), le cadre et les principales institutions juridiques des États dans le monde ont été implantés par le biais de la colonisation. Telle est la seconde thèse soutenue par cette théorie : Legal origin matters.

Ainsi, alors que la Law and Economics tentait d'établir le lien entre droit et économie et, plus précisément, de mesurer l'influence du droit sur l'économie, la Legal Origins Theory pose désormais que les institutions juridiques d'un État sont le produit d'une tradition juridique exportée par les grandes puissances colonisatrices. C'est, plus précisément, l'origine juridique d'un État qui aurait une influence sur sa croissance économique.

\subsection{Common Law vs Civil Law}

Quelles sont les différentes origines juridiques permettant de classifier les différents États à travers le monde ? L'exercice de classification a longtemps dominé l'histoire du droit comparé. L'un des premiers auteurs à se livrer à cet exercice fut A. Esmein qui, lors du Congrès international de droit comparé de 1905, répartit les ordres juridiques étatiques en cinq groupes (latin, germanique, anglo-saxon, slave et islamique $)^{10}$, sans toutefois préciser les critères pris en considération pour procéder à une telle classification ${ }^{11}$. D'autres suivirent, se fondant notamment sur des critères linguistiques et géographiques ou encore raciaux ${ }^{12}$. Plus tard, H. Lévy-Ullman proposa de distinguer les ordres juridiques en fonction des sources de droit ${ }^{13}$. Il fallut toutefois attendre le mitan du $\mathrm{xx}^{\mathrm{e}}$ siècle pour voir apparaître une classification fondée sur le fond du droit étudié. P. Arminjon, B. Nolde et M. Wolf proposèrent une des premières classifications modernes en sept familles juridiques ${ }^{14}$ :

9. R. Michaels, « Comparative Law by Numbers? Legal Origins Thesis, Doing Business Reports, and the Silence of Traditional Comparative Law », The American Journal of Comparative Law, vol. 57, 2009, pp. 769 et s.

10. A. Esmein, «Le droit comparé et l'enseignement du droit », in Congrès international de droit comparé, Procès-verbaux des séances et documents, Paris, 31 juillet au 4 août 1900, tome I, Paris, LGDJ, 1905, pp. 451-452.

11. P. Arminjon, B. Nolde et M. Wolf, Traité de droit comparé, Paris, LGDJ, 1950, p. 43.

12. G. Sauser-Hall propose une classification des systèmes juridiques fondée sur la race : la race aryenne ou indo-européenne, la race sémitique et les barbares (G. SAUSER-HALL, Fonction et méthode du droit comparé, Genève, Kundig, 1913).

13. H. LÉvy-UlLmanN, « Observations générales sur les communications relatives au droit privé dans les pays étrangers », in Les transformations du droit dans les principaux pays depuis cinquante ans, tome I, Paris, LGDJ, 1922, pp. 81 et s. Il y aurait ainsi trois grands groupes : les pays dits continentaux, de droit écrit, les pays de langue anglaise, pays de coutume (jurisprudentielle), et le droit de l'Islam qui se distinguerait par «son immobilité presque absolue » et son caractère religieux (ibid., pp. 86-87).

14. P. Arminjon et al. définissent la famille ou le système comme « un groupement de personnes unies par un ensemble de règles juridiques qui ordonnent soit tous les éléments, soit au moins les principaux 
française, germanique, scandinave, anglaise, russe, islamique et indu ${ }^{15}$. À peu près au même moment, R. David se fondait sur des critères idéologiques et, dans une moindre mesure, sur les techniques juridiques utilisées pour proposer une classification en cinq grands systèmes juridiques : occidental, socialiste, islamique, indu et chinois ${ }^{16}$. Par la suite, il s'est essentiellement concentré sur les trois systèmes qu'il juge principaux : le système romano-germanique, la famille de Common Law et le système socialiste ${ }^{17}$. Reprenant l'œuvre de R. David, C. Jauffret-Spinosi ${ }^{18}$ conserve une classification identique, mais remplace logiquement le système socialiste par le système russe. Enfin, dans la dernière version de leur introduction au droit comparé1920, K. Zweigert et H. Kötz, principaux inspirateurs du courant de la Legal Origins Theory, s'intéressent principalement à quatre grandes familles, chacune se distinguant par un $«$ style $»^{21}$ qui lui est propre ${ }^{22}$ : romaniste, germanique, scandinave et de Common Law $^{23}$.

En grande partie fondée sur cette dernière classification, la catégorisation de la Legal Origins Theory s'appuie essentiellement sur la dichotomie entre Common Law, d'une part, et Civil Law, d'autre part. Une distinction supplémentaire y est opérée, au sein de la famille civiliste, entre trois pôles d'influence : français, germanique et scandinave.

Le système de Civil Law se caractérise essentiellement par sa descendance $\mathrm{du}$ droit romain. La division entre droit romain et coutumes germaniques s'est estompée par la réception du droit romain en Allemagne et en Europe centrale, ce qui a conduit à l'élaboration d'un droit commun - d'un jus commune - en Europe continentale jusqu'à la fin du XVIII ${ }^{\mathrm{e}}$ siècle. Cette unité fut ensuite menacée, sans

éléments de leur vie sociale, et souvent aussi par des institutions juridictionnelles et administratives communes » (P. Arminjon, B. Nolde et M. Wolf, Traité de droit comparé, op. cit., p. 10). K. Zweigert et $\mathrm{H}$. Kötz, considèrent leur division comme la plus convaincante, car elle se fonde sur la substance du droit étudié et non pas sur un critère qui lui est extérieur (K. ZwEIGERT \& H. KöTZ, An Introduction to Comparative Law, 3rd ed., New York, Oxford University Press, 1998, p. 65).

15. P. Arminjon, B. Nolde et M. Wolf, Traité de droit comparé, op. cit., p. 47.

16. R. DAVID, Traité élémentaire de droit civil comparé : introduction à l'étude des droits étrangers et la méthode comparative, Paris, LGDJ, 1950, p. 224.

17. Tout en continuant à s'intéresser à quatre autres systèmes - qu'il classe dans le chapitre « Autres conceptions de l'ordre social et du droit »-que sont les systèmes de droit musulman, indu, d'Extrême-Orient et d'Afrique et de Madagascar (R. DAVID et C. JAUfFRET-SpInosi, Les grands systèmes de droit contemporain, $9^{\mathrm{e}}$ éd., Paris, Dalloz, 1988).

18. R. David et C. JAufFret-Spinosi, Les grands systèmes de droit contemporain, $11^{\mathrm{e}}$ éd., Paris, Dalloz, 2002.

19. K. Zweigert \& H. Kötz, An Introduction to Comparative Law, op. cit.

20. Dans la première version de leur ouvrage, ils distinguaient huit familles juridiques : la romaniste, la germanique, l'anglo-américaine, la nordique, la socialiste et, à titre résiduel, celles d'ExtrêmeOrient, de droit islamique et de droit indu.

21. Le concept de «style »-emprunté à la littérature et aux beaux-arts - se définit par cinq principaux traits : le contexte historique, le mode de pensée juridique, les institutions, les sources de droit et l'idéologie.

22. K. Zweigert \& H. Kötz, An Introduction to Comparative Law, op. cit., p. 67.

23. Les autres droits étudiés sont ceux d'Extrême-Orient (droit chinois et droit japonais) et les systèmes légaux religieux (islamique et indu). 
pour autant être détruite, par les codifications nationales du XIX $\mathrm{X}^{\mathrm{e}}$ siècle $^{24}$. Ces différentes codifications menèrent nécessairement vers une forme de scission ; néanmoins, par leurs sources d'inspiration, de grandes similitudes demeurèrent, de telle manière que l'on peut encore considérer à l'heure actuelle que ces différents systèmes font toujours partie de la même culture juridique. Le droit romain fut en effet la principale source utilisée pour l'élaboration des différents codes nationaux, tant par son contenu (la théorie des obligations en droit civil est restée commune à tous les pays du groupe romaniste) que par sa manière de penser le droit, puisqu'une science juridique commune s'était développée dans les universités européennes depuis le $\mathrm{XII}^{\mathrm{e}}$ siècle. En dépit de ces codifications et malgré de nombreuses spécificités propres à chaque législation nationale, le droit continental européen conserve donc, dans une appréhension globale, sa structure unifiée ${ }^{25}$.

Le deuxième grand système est celui de Common Law, d'origine anglaise. Le droit romain n'a jamais vraiment réussi à traverser la Manche, malgré la période de domination normande. En 1066, Guillaume le Conquérant sort vainqueur de la bataille de Hastings et marque le début de la conquête de l'Angleterre. Il confisque les terres appartenant aux nobles anglo-saxons pour les distribuer aux siens et instaure, de manière artificielle, la féodalité. Il met en place une Curia Regis compétente pour régler les questions de droit public, de propriété foncière (considérée comme relevant du domaine public dans le système féodal) et les crimes les plus graves. Les juges liés à la juridiction royale ne furent dès lors pas nourris de droit romain - celui-ci étant essentiellement de droit privé -, à l'inverse des juges continentaux. Pour s'adresser aux juges anglais, les particuliers devaient demander au Roi, plus précisément au Chancelier, la délivrance d'un $w^{2} i^{26}$ qui leur permettait de saisir exceptionnellement les juridictions royales. L'extension progressive de ces writs a permis aux juges royaux de constituer un droit nouveau qu'ils présentèrent comme la « coutume générale et immémoriale du Royaume ». C'est ainsi que s'est progressivement fondé le système de Common Law ${ }^{27}$, à l'origine essentiellement publiciste (c'est-à-dire de droit public), procédural et judiciaire ${ }^{28}$. Pour offrir une certaine stabilité à ce système dépourvu de loi écrite, les juges ont pris l'habitude de se conformer à la règle obligatoire du précédent. Ainsi, toute décision judiciaire s'impose non seulement au juge qui l'a rendu, mais également à tous les autres juges. Il était toujours permis, néanmoins, de s'adresser directement au Roi, ou plutôt au Chancelier, en vue d'obtenir une solution équitable. Le développement de la loi écrite a fini par accompagner la montée en puissance du Parlement au fil de l'histoire d'Angleterre. Le droit anglais a

24. L'objectif initial de la codification napoléonienne (la première en date) était pourtant de compiler un droit fondé sur la « Raison », par conséquent unique et traversant les frontières, mais le rejet de la codification française en Allemagne a mis fin au rêve d'un droit uniforme européen (R. DAVID et C. JAUfFret-Spinosi, Les grands systèmes de droit contemporain, $9^{e}$ éd., op. cit., p. 69).

25. M. Ancel, Utilité et méthodes du droit comparé, Neuchâtel, Éditions Ides et Calendes, 1971, pp. 44-45.

26. En fonction du writ, découlait une procédure particulière.

27. La « loi commune», par opposition aux coutumes locales.

28. M. Ancel, Utilité et méthodes du droit comparé, op. cit., p. 46. 
donc une structure tripartite, composée de la Common Law, c'est-à-dire le droit jurisprudentiel, de l'Equity, qui joue le rôle de correcteur, et du Statute Law, ensemble des lois écrites votées par le Parlement ${ }^{29}$. À l'inverse du Civil Law, au sein duquel la loi, règle de conduite abstraite, occupe une place prépondérante, la Common Law, formée essentiellement par les juges, vise principalement à donner une solution concrète à un procès ${ }^{30}$, donc à un cas particulier.

Selon les tenants de la Legal Origins Theory, ces deux grands systèmes se sont ensuite répandus dans le monde au moyen de politiques d'expansion et de colonisation $^{31}$.

À cet égard, en ce qui concerne la division tripartite au sein de la culture de Civil Law, il faut d'emblée rappeler que le Code de commerce français fut rédigé en 1807, sous le contrôle de Napoléon, qui l'imposa tel quel en Belgique, aux PaysBas, ainsi que dans une partie de la Pologne, de l'Italie et des régions occidentales de l'Allemagne. Il fut également implanté dans les différentes colonies françaises en Afrique du Nord, de l'Est et subsaharienne, en Océanie, en Indochine et dans les Caraïbes. La culture juridique française a également influencé de manière prépondérante le Luxembourg, le Portugal, l'Espagne et certains cantons suisses. Lors de la dissolution des empires espagnol et portugais en Amérique latine au XIX ${ }^{\mathrm{e}}$ siècle, les États émergents se sont principalement inspirés du système légal français ${ }^{32}$; il est vrai que ces États avaient déjà été influencés en ce sens par les colons espagnols et portugais, eux-mêmes imprégnés de la culture juridique française.

Le Code de commerce allemand eut un moins grand retentissement, peut-être dû à sa rédaction plus tardive ${ }^{33}$. Il n'en eut pas moins une grande influence sur les pays d'Europe centrale et orientale (Autriche, République tchèque, Slovaquie, exYougoslavie, Hongrie, Grèce ou Suisse), mais également sur des pays asiatiques, tels que le Japon ou la Corée.

La culture scandinave, dont les liens politiques et culturels ont toujours été très proches $^{34}$, se cantonne aux quatre États qui la constituent : le Danemark, la Suède, la Norvège et la Finlande.

29. Ibid., p. 47.

30. R. David et C. Jauffret-Spinosi, Les grands systèmes de droit contemporain, 9e éd., op. cit., p. 22.

31. R. La Porta, F. Lopez-de-Silanes, A. Shleifer \& R. Vischny, «Law and Finance », Journal of Political Economy, vol. 106, 1998, pp. 1117-1121. Cette thèse n'est toutefois pas novatrice et s'appuie sur les travaux antérieurs de comparatistes (voy. not. K. Zweigert \& H. Kötz, An Introduction to Comparative Law, op. cit., pp. 65-67, 109 et 219 ; R. DAVID et C. JAUfFret-SPINOSI, Les grands systèmes de droit contemporain, $9^{e}$ éd., op. cit., pp. 24, 25, 78 et s., 638 et s.).

32. Le droit français était le plus familier des droits portugais et espagnol - qui ne pouvaient bien évidemment plus être le modèle juridique à suivre - et était le produit de la Révolution française, modèle d'inspiration pour ces nouveaux États indépendants (K. ZwEIGERT \& H. KÖTz, An Introduction to Comparative Law, op. cit., pp. 113-115).

33. Le Code général de commerce allemand (das Allgemeine Deutsche Handelsgesetzbuch) fut rédigé en 1861.

34. K. Zweigert \& H. Kötz, An Introduction to Comparative Law, op. cit., p. 277. 
Quant à la Common Law, elle s'est propagée au large des nombreuses colonies britanniques, telles que, entre bien d'autres, les États-Unis, le Canada, l'Australie, l'Inde.

Si, dans la plupart des cas, la classification n'est pas controversée, certaines situations sont toutefois plus complexes. Ainsi, le droit japonais, à l'origine inspiré du droit allemand, fut fortement influencé par le droit américain au lendemain de la Seconde Guerre mondiale ${ }^{35}$. Néanmoins, malgré l'existence de statuts plus « hybrides », les tenants de la Legal Origins Theory font le choix de l'origine plutôt que des révisions ultérieures, considérant l'ancrage d'origine comme plus profond.

\section{LA LEGAL ORIGINS THEORY}

La Legal Origins Theory a originellement pris forme à la fin des années 1990 au sujet du droit des sociétés. À la suite d'une série d'études empiriques, des chercheurs américains sont arrivés à la conclusion que les actionnaires minoritaires - voire les investisseurs en général - bénéficient d'une meilleure protection dans les pays d'influence anglo-saxonne, ce qui entraîne, d'une part, une plus grande dispersion de l'actionnariat et, par voie de conséquence, une gestion plus efficiente des entreprises et, d'autre part, un développement des marchés financiers et un meilleur accès pour les entreprises aux sources de financement externe - tant sous forme de prêt que de capital à risque.

Quatre chercheurs sont à l'origine de cette théorie: Raphael La Porta, Florencio Lopez-de-Silanes, Andrei Shleifer (tous trois professeurs à l'Université de Harvard) et Robert W. Vishny (professeur à l'Université de Chicago), dont les initiales de chacun forment l'acronyme «LLSV », appellation adoptée dans la littérature spécialisée pour désigner les auteurs fondateurs de la Legal Origins Theory.

Dans une première étape, nous analyserons le domaine dans lequel la Legal Origins Theory a éclos, c'est-à-dire le droit des sociétés, à travers l'attention accordée à la protection des investisseurs. Nous nous référerons principalement à trois études considérées comme fondatrices en la matière : « Law and Finance $»^{36}$, «Corporate Ownership Around the World $»^{37}$ (à laquelle R. Vishny n'a pas participé $^{38}$ ) et "Legal Determinants of External Finance $»^{39}$ (3.1.). Dans une seconde

35. Pour plus de développements, voy. J.-L. Halperin, « Le Code de commerce au Japon : une brève histoire ou le code sans esprit », 2009, disponible sur : https://halshs.archives-ouvertes.fr/ halshs-00387039.

36. R. La Porta, F. Lopez-de-Silanes, A. Shleifer \& R. Vischny, «Law and Finance », op. cit., note 31, pp. 1113-1155.

37. R. La Porta, F. Lopez-de-Silanes \& A. Shleifer, « Corporate Ownership Around the World », The Journal of Finance, vol. 54, 1999, pp. 471-517.

38. Nous utiliserons dès lors l'acronyme « LLS », également utilisé par la doctrine.

39. R. La Porta, F. Lopez-de-Silanes, A. Shleifer \& R. Vischny, « Legal Determinants of External Finance », The Journal of Finance, vol. LII, n³, 1997, pp. 1131-1150. 
étape, nous déterminerons comment cette théorie s'est étendue à d'autres domaines. De nombreuses études empiriques - menées notamment par LLSV, mais également par d'autres chercheurs - ont en effet appliqué le critère de la Legal Origin pour analyser différentes réglementations juridiques et mesurer leur impact sur l'économie (3.2.). Cette théorie ne s'est toutefois pas cantonnée au milieu de la recherche. Elle a fortement influencé les rapports Doing Business de la Banque mondiale, en particulier leurs premières versions, dont l'objectif est de poser des recommandations plus que de simples observations (3.3.).

\subsection{Son lieu de naissance : le droit des sociétés}

La Legal Origins Theory est donc née d'une réflexion sur le droit des sociétés et c'est à ce lieu de naissance que nous allons nous intéresser. Les tenants de la théorie commencent par remettre en cause le caractère universel du modèle d'actionnariat dispersé, tel que décrit par Berle et Means, mais considèrent que cette description est le propre des sociétés agissant dans un cadre juridique d'influence anglosaxonne (3.1.1.). Selon ces mêmes auteurs, cette structure de l'actionnariat serait la conséquence du haut niveau de protection accordé aux investisseurs (3.1.2.). Or cette même protection influencerait également le développement des marchés financiers et, par conséquent, l'accès aux sources de financement externe pour les entreprises (3.1.3.).

\subsubsection{Remise en cause d'un régime universel de l'actionnariat dispersé}

Dans leur ouvrage de référence The Modern Corporation and Private Property ${ }^{40}$, Berle et Means observent la scission entre propriété et gestion de l'entreprise. En raison de leur croissance, les grandes sociétés américaines seraient devenues des Management Corporations, dont la taille désormais hégémonique entraîne un degré de complexité provoquant un déplacement de l'exercice du pouvoir des actionnaires vers les dirigeants : une scission s'opère dès lors entre propriété et exercice du pouvoir. Les actionnaires, petits porteurs dispersés, délèguent leurs pouvoirs aux gestionnaires en échange d'un dividende stable. S'ils n'approuvent pas la gestion de l'entreprise, ils ont intérêt à vendre leurs actions plutôt qu'à contester le management. De cette description de la société moderne ou plutôt d'un certain type de société moderne - puisque, comme nous le verrons, elle est spécifique au système anglo-américain -, une majorité de la doctrine anglo-saxonne déduit qu'il s'agit du modèle à suivre. Elle opère ainsi un glissement du point de vue descriptif au niveau prescriptif ${ }^{41}$.

40. A.A. Berle Jr. \& G.C. Means, The Modern Corporation and Private Property, New York, Harcourt, Brace and World, 1992 (rééd.).

41. A. Autenne, «Analyse économique des offres publiques d'achat », in Y. De Cordt, P. LAmbrecht et P. HAMer (dir.), La réforme de la réglementation sur les offres publiques d'achat, Bruxelles, Kluwer, 2007, p. 354. 
Dans «Corporate Ownership Around the World ${ }^{42}$, LLS analysent le régime de propriété des sociétés au sein d'une série d'États et mettent d'emblée à mal le modèle général de Berle et Means : l'image d'un actionnariat dispersé est en fait l'exception dans la majorité des États. Cette configuration est en réalité, selon les auteurs, la conséquence d'un régime juridique favorable aux investissements, principalement grâce à une bonne protection des actionnaires minoritaires. De ce point de vue, une corrélation positive existerait entre le degré de protection des investisseurs et l'appartenance à une culture juridique déterminée. En l'occurrence, l'appartenance à la famille de Common Law serait synonyme d'une bonne protection des investisseurs et, dès lors, d'une situation favorable à la dispersion de l'actionnariat.

Dans leur étude, LLS prennent pour échantillon les plus grandes entreprises cotées en bourse des vingt-sept plus grandes économies de l'époque, c'est-à-dire du début des années 1990. Si le choix porte sur les plus grandes sociétés, c'est parce que les chercheurs s'appuient sur l'hypothèse - qui sera vérifiée par leurs résultats empiriques - qu'une société plus large est davantage susceptible de détenir un actionnariat dispersé.

La base de données comprend donc les vingt-sept États les plus riches en fonction de leur revenu par habitant - per capita income - en 1993, à l'exclusion de certains ne détenant pas un assez grand marché boursier (tels que le Koweït, les Émirats arabes unis ou l'Arabie saoudite par exemple) ${ }^{43}$. Pour chaque État, deux échantillons de sociétés sont pris en compte. Le premier reprend les vingt plus grandes sociétés selon leur indice de capitalisation boursière à la fin de l'année 1995. Ce critère pose néanmoins un problème d'envergure quant à l'objectivité d'une comparaison entre différents États. Les vingt plus grandes sociétés belges, par exemple, sont en effet bien plus petites que les vingt plus grandes sociétés américaines et sont dès lors moins susceptibles d'être détenues par un actionnariat éparpillé. C'est la raison pour laquelle un deuxième échantillon s'imposait : celui-ci reprend les dix plus petites sociétés ayant un indice de capitalisation boursière d'un minimum de 500 millions de dollars, autrement dit les dix sociétés dont l'indice se rapproche le plus possible de 500 millions de dollars sans être inférieur à ce montant. Cette seconde base permet donc de comparer des grandes sociétés de taille similaire et est ainsi plus appropriée à une approche comparative du régime de propriété des sociétés d'un État à l'autre.

Afin d'observer la concentration de propriété, le point de vue adopté est celui des droits de vote (voting rights) plutôt que celui des droits aux dividendes (cash

42. R. La Porta, F. Lopez-de-Silanes \& A. Shleifer, « Corporate Ownership Around the World », op. cit., note 37 .

43. La liste des différents États est la suivante : Allemagne, Argentine, Australie, Autriche, Belgique, Canada, Corée du Sud, Danemark, Espagne, Finlande, France, Grèce, Hong Kong, Irlande, Israël, Japon, Mexique, Nouvelle-Zélande, Norvège, Portugal, Singapour, Suède, Suisse, RoyaumeUnis, États-Unis. 
flow rights). La raison de ce choix est liée au questionnement de Berle et Means, qui cherchaient à savoir qui, des actionnaires ou des gestionnaires, contrôle la société. Puisqu'il est question de contrôle, il est donc davantage intéressant de s'attarder sur les droits politiques plutôt que sur les droits économiques. Pour analyser cette concentration en termes de contrôle, deux seuils sont utilisés : le premier est fixé à $10 \%$ des droits de vote - seuil considéré comme significatif d'un grand degré de contrôle et dont le dépassement entraîne généralement une obligation de notification aux autorités publiques - ; le second est porté à $20 \%$ - taux considéré comme suffisant pour contrôler effectivement une société.

En analysant les plus grandes sociétés, c'est-à-dire le premier échantillon, et en prenant en compte le critère de détention de $20 \%$ des droits de vote pour obtenir le contrôle effectif, seulement $36 \%$ des sociétés sondées apparaissent dotées d'un actionnariat dispersé et n'ont dès lors pas d'actionnaire de contrôle. En ce qui concerne le deuxième panel d'entreprises - celles dont la capitalisation boursière est de 500 millions de dollars au minimum tout en se rapprochant le plus de ce montant -, ce chiffre n'est plus que de $23 \%$ - ce qui confirme la présomption quant à la corrélation positive entre grandeur d'une société et dispersion de son actionnariat.

Parmi les sociétés soumises à l'influence déterminante d'un «propriétaire », celui-ci se matérialise le plus souvent sous la forme de l'État ou d'une famille, plutôt que sous celle d'une autre société ou d'une institution financière. L'utilisation du terme « propriétaire » (owner) diffère de son sens classique : le mot est ici utilisé pour désigner un actionnaire qui détient un pourcentage suffisant de droits de vote pour contrôler effectivement la société, à savoir $20 \%$. Il est parfois nécessaire de remonter la structure pyramidale composée de sociétés intermédiaires pour découvrir ce propriétaire final. À titre d'illustration, si une société A détient $20 \%$ des droits de vote de la société $\mathrm{B}$, qui possède elle-même $20 \%$ des droits de vote de la société $\mathrm{C}$, il s'agit alors d'une structure pyramidale au sein de laquelle A est le «propriétaire final» (ultimate owner) de la société C. La fréquence à laquelle l'État se retrouve actionnaire majoritaire d'une société s'expliquerait par le non-aboutissement des mécanismes de privatisation dans la majorité des économies - c'était en tout cas la constatation de 1999 - ainsi que par la montée en puissance de l'État-Providence au lendemain de la Seconde Guerre mondiale. Le résultat est néanmoins plus surprenant en ce qui concerne la propriété familiale : elle constitue $30 \%$ des sociétés du premier panel, c'est-à-dire des plus grandes sociétés, et $45 \%$ du second, c'est-à-dire des sociétés dont l'indice de capitalisation boursière se rapproche de 500 millions de dollars sans être inférieur à ce montant. Or, lorsqu'une société est sous un régime de propriété familiale, dans $69 \%$ des cas, un des membres de la famille participe également à la gestion de l'entreprise (en tant que Chief Executive Officer, par exemple). Cette situation pose un problème, car elle expose les actionnaires minoritaires au risque d'expropriation, c'est-à-dire l'appropriation des ressources par les actionnaires majoritaires au détriment des 
minoritaires, à tout le moins s'ils ne bénéficient pas de bonnes protections légales. L'expropriation des actionnaires minoritaires peut se matérialiser de nombreuses manières, notamment via des prix de transfert, la vente d'actifs entre sociétés d'un même groupe ou bien encore par l'intermédiaire d'augmentations de capital dilutives ou réservées par exemple ${ }^{44}$. L'existence d'un autre actionnaire important au sein de l'entreprise serait une manière de pallier ce risque, car elle créerait une situation analogue à celle du dilemme du prisonnier ${ }^{45}$ : chacun des principaux actionnaires ne peut se lancer dans un processus d'expropriation discrétionnaire sans craindre une contre-réaction de l'autre actionnaire ; cet équilibre des forces installerait un contrôle mutuel et jouerait donc à l'avantage des investisseurs minoritaires, lesquels ne devraient plus être inquiétés par une éventuelle expropriation. Malheureusement, dans $75 \%$ des cas, aucun actionnaire n'est suffisamment grand pour jouer ce rôle de surveillance.

Dans leur analyse, LLS constatent également que les grands actionnaires ont un pouvoir de contrôle qui excède la proportion de leurs droits économiques (cash flow rights). Cette disproportion résulte du fait que, d'une part, les propriétaires jouent souvent un véritable rôle dans la gestion de l'entreprise et, d'autre part, que les sociétés sont souvent contrôlées par l'intermédiaire de structures pyramidales. La détention en cascade de participations via des structures juridiques de détention de capital (holdings), dont l'actionnaire conserve le contrôle majoritaire, permet en effet de bénéficier d'une puissance de vote démultipliée, permettant de conserver le contrôle au moyen d'une proportion relativement faible d'investissements. L'instauration du principe one share, one vote - c'est-à-dire la parfaite adéquation entre droits politiques et droits économiques - n'est pas de nature à bouleverser la donne, car c'est essentiellement par le biais de la structure pyramidale que les actionnaires séparent la propriété du contrôle.

Cette étude conduit donc LLS à conclure que l'analyse de Berle et Means est loin d'être universelle. Elle décrit avec justesse le système anglo-américain et, par extension, celui des États appartenant à la famille de Common Law, mais cette situation est loin de s'imposer comme le principe à l'échelle mondiale. Se pose dès lors la question de la raison de cette dispersion de l'actionnariat, si particulière à la culture anglo-américaine.

44. P. Navatte et G. Schier, «Le “Tunneling”. Ou l'expropriation des actionnaires minoritaires : l'exemple des augmentations de capital réservées », Gestion 2000, 2011/1, p. 46.

45. Cette expression fait référence à la théorie exposée par A.W. Tucker en 1950, originellement illustrée par la situation suivante : deux délinquants sont arrêtés pour un délit et sont placés dans des cellules distinctes sans qu'ils aient eu la possibilité de se consulter préalablement. Si l'un choisit de dénoncer l'autre, il ne sera pas poursuivi, mais son témoignage sera utilisé pour condamner son acolyte à trois ans de prison. S'ils se dénoncent mutuellement, ils seront tous deux condamnés à deux ans de prison. S'ils gardent le silence, par contre, ils ne se verront infliger qu'une année de prison (E. Mackay et S. Rousseau, Analyse économique du droit, Paris, Dalloz, 2008, p. 45). Bien que la solution la plus avantageuse pour chaque prisonnier soit de trahir l'autre sans être luimême trahi, l'attitude la plus raisonnable est d'éviter tout comportement hostile puisque chacun n'a aucune marge de manœuvre ni information sur la décision de l'autre. Cette situation tend donc vers un équilibre des forces. 
La réponse se trouverait du côté du degré de protection accordé aux investisseurs. En effet, les sociétés opérant dans un cadre juridique moins protecteur à l'égard des investisseurs tendraient vers une plus forte concentration de leur actionnariat. Ce processus pourrait s'expliquer par deux raisons au moins. Premièrement, sans grande protection, la volonté de l'investisseur d'accroître ses droits afin d'éviter l'expropriation du pouvoir par les managers se matérialiserait par l'acquisition de capital supplémentaire. Deuxièmement, dépourvues de protection, les actions et obligations seraient moins attractives et les épargnants ne seraient donc guère disposés à en acquérir, si ce n'est à un prix tellement bas que les sociétés n'auraient pas d'intérêt à émettre de nouvelles actions sur le marché.

Il se trouve que la protection accordée aux investisseurs, particulièrement aux actionnaires minoritaires, serait la plus forte dans les systèmes juridiques d'origine anglo-saxonne. Une deuxième étude ${ }^{46}$ - chronologiquement la première - menée par le même collectif d'auteurs, auquel s'ajoute la participation de R. Vishny, s'intéresse de manière comparative au degré de protection accordé aux investisseurs par différents États. Il convient d'en présenter synthétiquement les résultats dans les lignes qui suivent afin de compléter la présentation du raisonnement de LLSV.

\subsubsection{Influence des origines juridiques d'un État sur la protection des investisseurs}

Cette deuxième étude empirique porte sur quarante-neuf États à travers le monde, le panel ne comprenant aucune économie socialiste, ni de transition (pour rappel, nous sommes alors en 1998). Ces États sont groupés en fonction de l'origine de leur système juridique. Comme nous l'avons indiqué précédemment ${ }^{47}$, selon les tenants de la Legal Origins Theory, même si chaque système légal a ses spécificités, un degré de similitude persiste et permet de les classer selon des ensembles plus globaux, c'est-à-dire selon des familles juridiques. Les droits des différents États n'ont pas émergé de nulle part, leur base résulte d'une transposition du cadre juridique de la culture à laquelle ils appartiennent. Si chaque système juridique étatique a fait l'objet d'une évolution, synonyme de spécification, il n'en reste pas moins qu'une appartenance à l'une des deux grandes cultures juridiques - Common Law, d'une part, et Civil Law, d'autre part - demeure. Cela permet une comparaison précise entre chaque État, mais également une comparaison plus globale entre une manière anglo-saxonne et une manière disons continentale de penser le régime juridique de la protection des investisseurs.

Grâce à la classification opérée au départ du raisonnement ${ }^{48}$, les systèmes juridiques des États étudiés peuvent être comparés sous l'angle du droit des sociétés dans quatre domaines significatifs : les droits des actionnaires (3.1.2.1.), les

46. R. La Porta, F. Lopez-de-Silanes, A. Shleifer \& R. Vischny, « Law and Finance », op. cit., note 31, pp. 1113-1155.

47. Cf. supra point 2.2.

48. Idem. 
droits des créanciers (3.1.2.2.), l'effectivité de la protection accordée (3.1.2.3.) et la concentration de propriété (3.1.2.4.).

\subsubsection{Droits des actionnaires}

Pour évaluer la protection accordée aux actionnaires, LLSV élaborent un indicateur-dénommé Antidirector Rights Index - qui compare les différents États sur la base de six critères. Le premier critère, appliqué dans seulement onze des États sondés, est le principe one share, one vote, dont l'objectif est d'obtenir une parfaite adéquation entre les parts détenues et les droits politiques qui en découlent, dans le dessein d'éviter qu'un actionnaire ne dispose d'un pouvoir disproportionné par rapport à sa quote-part dans le capital de l'entreprise. Le deuxième critère consiste en la possibilité de voter par courrier électronique, ce qui facilite notamment la participation des petits actionnaires qui ne prennent pas le temps de se déplacer pour participer à l'assemblée générale ${ }^{49}$. Le troisième est la liberté pour l'actionnaire de ne pas devoir déposer ni bloquer ses actions dans une période précédant le déroulement de l'assemblée générale à laquelle il souhaite assister. Le quatrième consiste en l'instauration d'un mécanisme de représentation proportionnelle, qui permet aux actionnaires minoritaires de nommer un nombre proportionnel d'administrateurs. Le cinquième critère est la proportion de capital nécessaire pour la convocation d'une assemblée générale extraordinaire. Le sixième et dernier consiste dans l'évaluation des mécanismes légaux destinés à contrer la mainmise des dirigeants au détriment des actionnaires minoritaires. Les exemples cités pour l'évaluation de ce critère sont la faculté de contester en justice les décisions des directeurs et le droit au rachat forcé en cas de désaccord avec des décisions fondamentales du management. Cette catégorie est bien plus large que les cinq autres, bien qu'elle fasse l'objet, à l'instar des autres critères, d'une évaluation binaire : soit l'État remplit la condition, soit il ne la remplit pas, sans place pour une évaluation intermédiaire. Alors que le choix même des critères peut déjà faire l'objet de discussions, l'évaluation de ce dernier offre un espace de subjectivité discutable pour une étude qui se prétend objective. Enfin, un dernier critère est pris en compte, mais il n'est pas mis sur un pied d'égalité avec les autres. Il s'agit du dividende obligatoire, considéré comme un substitut au déficit de protection accordé aux actionnaires.

Après avoir analysé la situation de chaque État à l'aune de chacun de ces critères, les auteurs en arrivent à la conclusion que les États appartenant à la famille de Common Law offrent une plus grande protection aux actionnaires minoritaires, alors que les systèmes juridiques d'origine française et allemande offrent la protection la plus faible. Entre ces deux groupes, les pays scandinaves se caractériseraient par un degré de protection moyenne. Les dividendes ne sont obligatoires que dans certains pays de Civil Law - ce qui attesterait qu'il s'agit bien là d'un substitut à

49. Voy., à cet égard, pour les États membres de 1'Union européenne, la directive 2007/36/CE du Parlement européen et du Conseil du 11 juillet 2007 concernant l'exercice de certains droits des actionnaires de sociétés cotées, adoptée dans le but de redynamiser les assemblées générales. 
une faible protection. Il est néanmoins intéressant de s'interroger sur la qualification de critère de substitution. Le droit au dividende obligatoire est certes un droit à caractère économique et non politique, mais il s'agit d'une véritable protection de l'actionnaire quant à son retour sur investissement. Bien que le critère ait été considéré comme de substitution avant l'analyse des résultats, nous ne serions pas étonnés d'apprendre que cette qualification ait été réalisée a posteriori, afin de conforter le présupposé sous-jacent.

LLSV anticipent judicieusement la critique qui consiste à affirmer qu'une solide protection actionnariale ne serait pas due à l'origine juridique des États, mais à leur richesse. Ils ont donc réorganisé le panel, en le divisant en trois groupes en fonction de leur produit national brut ${ }^{50}$. Face à cette nouvelle disposition, ils détectent certes une corrélation positive entre richesse et protection de l'actionnariat, mais celle-ci est nettement moins significative que l'origine juridique.

\subsubsection{Droits des créanciers}

La situation est un peu plus complexe sous l'angle de la protection des créanciers, car il existe plusieurs types de créanciers. Dès lors, la protection d'une certaine catégorie se fait potentiellement au détriment d'une autre. En effet, en situation de concours, le principe d'égalité des créanciers impose que le prix de réalisation des biens du débiteur soit réparti entre les différents créanciers de manière proportionnelle, chacun étant donc payé en fonction du quantum de sa créance. Néanmoins, il est possible - et même probable - que l'un d'eux possède une cause de préférence et rompe donc le principe d'égalité, alors même que favoriser une catégorie de créanciers se fait nécessairement aux dépens des autres.

La deuxième difficulté provient du dilemme entre liquidation et réorganisation de l'entreprise. Le droit d'un créancier est naturellement de pouvoir récupérer sa créance lorsque le débiteur est en défaut de paiement, mais, dans certaines situations, ce paiement forcé entraînerait la liquidation de la société débitrice, conséquence socialement indésirable. Dès lors, certains États suspendent l'exécution forcée afin de laisser un délai au débiteur pour permettre à l'entreprise de se réorganiser.

L'étude se borne à l'évaluation binaire de quatre critères et se focalise en outre sur le pourcentage de capital exigé pour former une réserve obligatoire, qui constitue, à l'instar du dividende obligatoire pour les droits des actionnaires, un critère de substitution. Nous allons encore une fois énumérer les différents critères pris en compte afin d'avoir une idée de ce que représente, pour LLSV, l'idéal de protection des créanciers. Le premier critère est celui de la non-cristallisation automatique de l'actif - situation de blocage qui empêche les créanciers prioritaires d'être payés par préférence, à l'avantage des gestionnaires et créanciers chirographaires.

50. Un groupe reprenant le quart des États les plus riches, un reprenant le quart des plus pauvres et enfin un dernier reprenant les $50 \%$ du milieu. 
Le deuxième, assez lié, est le fait que les créanciers préférentiels soient payés en premier. La troisième question qu'ils se posent est de savoir s'il y a des conditions à l'ouverture d'une procédure en réorganisation de l'entreprise ou si, au contraire, le gestionnaire jouit de la faculté de l'enclencher unilatéralement, sans le consentement des créanciers. Le dernier critère consiste à observer si, lors de l'ouverture d'une procédure en réorganisation, la gestion est retirée des mains de la direction pour être confiée à une tierce personne, désignée par les créanciers ou par une institution judiciaire. La mesure de substitution, pendant du dividende obligatoire, consiste donc dans l'obligation de constitution d'une réserve, amortissant la chute des créanciers en cas de défaut de paiement ${ }^{51}$. La somme de ces différents critères forme le Creditor Rights Index, permettant d'évaluer le degré de protection juridique accordé aux créanciers.

Une fois le système de protection des créanciers analysé dans chaque État à l'aune de ces différents critères, les chercheurs de l'étude en arrivent à la même conclusion: "The legal origin matters » ${ }^{52}$. Le schéma reste plus ou moins semblable : les systèmes juridiques d'origine anglo-saxonne sont les plus protecteurs, ceux d'origine française les moins protecteurs, tandis que les systèmes scandinaves et, cette fois, d'influence germanique se situent à mi-chemin, avec un léger avantage, en l'occurrence, pour les systèmes de type allemand. Étonnamment, le niveau de richesse, sans pour autant être un facteur déterminant, est corrélé négativement avec le degré de protection des créanciers. En effet, sans s'y étendre, les auteurs remarquent que les droits des créanciers sont plus forts dans les pays les plus pauvres, l'hypothèse la plus plausible étant le manque d'opportunités quant à d'autres types de financement.

À l'exception du régime allemand, se dégage donc une corrélation positive entre protection des actionnaires et protection des créanciers. L'origine juridique a donc une influence générale sur la protection des investisseurs. Les pays de Civil Law compensent néanmoins la fragilité de leur protection par des mécanismes de compensation, tels que les dividendes obligatoires ou les réserves légales.

\subsubsection{Effectivité de la protection}

LLSV passent ensuite à l'analyse de ce qu'ils appellent l'enforcement, en d'autres termes la mise en application (ou en vigueur) du système juridique, c'est-à-dire à l'étude de l'effectivité de la protection accordée dans chaque système, garantie par des mécanismes juridictionnels adéquats. Ils considèrent cette catégorie comme un substitut aux défaillances ou aux insuffisances des garanties légales. Pour reprendre leurs propres termes, "active and well-functioning courts can step in and rescue

51. Sur cette qualification de critère de substitution, nous réitérons la remarque préalablement formulée à l'égard du dividende obligatoire.

52. R. La Porta, F. Lopez-de-Silanes, A. Shleifer \& R. Vischny, «Law and Finance », op. cit., note 31 , p. 1138. 
investors abused by the management $\gg^{53}$. Les résultats placent cette fois-ci les pays scandinaves au premier plan, suivis de près par les systèmes d'influence allemande. Les systèmes de tradition française se retrouvent une fois encore en dernière position, devancés de peu par les systèmes de Common Law.

Les cinq critères utilisés sont les suivants : l'efficacité du système judiciaire, la suprématie de la rule of law (expression assez large que l'on pourrait résumer par le respect accordé au droit), le risque d'expropriation du gouvernement par le biais de la confiscation ou de la nationalisation, la possibilité de dénonciation d'un contrat par le gouvernement et, enfin, la qualité des normes comptables (la comptabilité joue un rôle essentiel puisqu'il s'agit du meilleur outil d'information dont disposent les investisseurs; la comptabilité financière ${ }^{54}$ est en effet destinée à rendre compte, de façon synthétique, de la performance économique d'une entreprise aux principaux utilisateurs externes, tels que les actionnaires et les créanciers ${ }^{55}$ ).

En ce qui concerne l'enforcement, contrairement à la protection des créanciers, le niveau de revenu par habitant a une grande influence sur la qualité de la mise en application des normes protectrices. Néanmoins, il s'agit d'un facteur supplémentaire car l'origine juridique joue toujours, selon LLSV, un rôle prépondérant.

\subsubsection{Concentration de la propriété}

La concentration de la propriété est également considérée comme un substitut au défaut de protection des investisseurs. Même si, sous cet angle, cette étude est bien moins approfondie que la première que nous avons analysée, il nous semble tout de même intéressant d'observer les chiffres obtenus, dans une perspective de comparaison.

LLSV prennent pour échantillon les dix plus importantes sociétés privées de chaque État, au sein desquelles ils observent le pourcentage détenu par les trois plus grands actionnaires. Les résultats montrent que les pays de tradition française ont tendance à une plus grande concentration de l'actionnariat. La moyenne des pourcentages de parts détenues par les trois plus grands actionnaires de chaque société s'élève en effet à $54 \%$. Les sociétés s'épanouissant dans un cadre juridique d'influence germanique et scandinave ont l'actionnariat le plus dispersé (avec respectivement une moyenne de 34 et $37 \%$ ). Le taux particulièrement bas au sein des systèmes d'origine germanique est justifié par la prise en compte des États asiatiques (Japon, Corée du Sud, Taiwan). Les États de Common Law, avec un pourcentage de $43 \%$, arrivent en deuxième position. Néanmoins, les pourcentages

53. Ibid., p. 1140.

54. La doctrine distingue généralement - malgré la porosité des frontières qui séparent ces deux notions - la comptabilité financière, destinée aux utilisateurs externes, de la comptabilité de gestion, destinée aux dirigeants et interne à l'entreprise.

55. H. Stolowy, M. Lebas, Y. Ding et G. Langlois, Comptabilité et analyse financière, une perspective globale, $3^{\mathrm{e}}$ éd., Bruxelles, De Boeck, 2013, p. 28. 
relatifs aux États-Unis et au Royaume-Uni sont particulièrement bas, avec une moyenne respective de 20 et $19 \%$.

La lecture combinée des deux articles souligne un raisonnement intéressant. Dans un premier temps, la théorie de Berle et Means est remise en cause, à tout le moins son caractère universel, puisqu'elle reflète principalement le cadre socioéconomique anglo-américain. Ensuite, il est apparu que l'éparpillement actionnarial serait la conséquence d'un cadre propice à l'investissement, où actionnaires et créanciers bénéficieraient d'une solide protection, laquelle serait la conséquence de l'appartenance d'un État à une culture juridique déterminée. La boucle est ainsi bouclée : ce qui apparaissait comme une analyse descriptive au premier abord devient en fin de compte le modèle à suivre, puisque la structure capitalistique des sociétés anglo-saxonnes est considérée comme la conséquence d'un système financier protecteur et efficace, dû à l'appartenance des États auxquels ressortissent ces sociétés à des systèmes de Common Law.

Précisons enfin que cette discussion, analysée du point de vue de la protection des investisseurs, peut également être reprise sous l'angle des modes de " gouvernance » (pour reprendre un terme à la mode) des entreprises et des modalités d'exercice du contrôle sur celle-ci ${ }^{56}$. L'analyse économique du droit en matière de gouvernance s'articule principalement, en effet, autour de la distinction entre le système anglo-américain (outsider ou arms lenght) et le système continental européen (insider ou control oriented). Comme son nom l'indique, le système angloaméricain s'appuie sur un mécanisme de contrôle externe de la gouvernance, plus précisément sur les marchés financiers. Comme nous l'avons souligné, les marchés de type anglo-saxon sont caractérisés par une grande liquidité des titres et par une dispersion de l'actionnariat. La stratégie se concentre dès lors sur la maximisation des cours boursiers afin de rendre l'entreprise plus attrayante et d'ainsi pouvoir augmenter sa capitalisation. La maximisation du cours est évidemment bénéfique pour les actionnaires puisque celle-ci gonfle la valeur de leur portefeuille, mais également pour les gestionnaires puisqu'elle entraîne une diminution du risque d'OPA, souvent synonyme de changement d'équipe de direction. Ce contrôle externe permet dès lors de diminuer les coûts d'agency, c'est-à-dire ceux résultant d'intérêts divergents entre actionnaires et gestionnaires. Le système insider d'Europe continentale est quant à lui fondé sur des modes de contrôle de la gouvernance internes à l'entreprise. La concentration de propriété, donc l'existence d'actionnaires majoritaires, en est un exemple. En effet, l'actionnaire majoritaire se retrouve en quelque sorte bloqué dans sa situation : s'il décide de se séparer d'une grande partie de ses titres, cela fera baisser le cours et sera interprété comme un signal de mauvais augure pour les investisseurs actuels ou potentiels. Voué à une certaine permanence, il sera nécessairement plus impliqué, alors, dans la gouvernance de l'entreprise.

56. A. Autenne, «Analyse économique des offres publiques d'achat », op. cit., pp. 341 et s. 
Une large partie de la doctrine en droit des sociétés prône un principe de convergence afin d'atteindre un modèle unique de contrôle de la gouvernance. Or le modèle qui remporte la préférence de la majorité des auteurs est celui de type outsider. Malgré le chemin différent, l'issue reste la même, c'est-à-dire la supériorité supposée du modèle de l'actionnariat dispersé. Si le processus de convergence ne s'opère pas naturellement vers le modèle outsider, ce n'est pas parce qu'il est inefficace, mais parce qu'il est conditionné par l'environnement juridique qui l'entoure. Ce modèle ne peut pleinement s'épanouir que dans un cadre légal protecteur des actionnaires minoritaires, c'est-à-dire principalement dans le cadre anglo-saxon. Le système légal a donc toute son importance : une fois encore, Law matters.

\subsubsection{Influence des origines juridiques sur le développement des marchés financiers}

Après s'être intéressés à la protection accordée aux investisseurs dans les deux grandes cultures juridiques et à son effet sur la dispersion de l'actionnariat, les auteurs interrogent désormais l'influence de cette protection - et donc également de l'origine juridique des États - sur la taille et l'importance des marchés de capitaux et, par conséquent, sur la capacité des sociétés à se financer, tant par crédit que par capital à risque ${ }^{57}$.

D'un point de vue méthodologique, les auteurs reconnaissent la difficulté de mesurer la taille d'un marché financier, dont les valeurs sont dominées par les plus grosses entreprises. Par ailleurs, les auteurs limitent leur étude à la corrélation entre marchés financiers et environnement juridique, sans évaluer les effets d'un tel marché sur l'économie - ce qui leur sera reproché ${ }^{58}$.

Pour calculer l'accès au financement par capital à risque, LLSV utilisent trois mesures. Premièrement, ils observent la capitalisation du marché boursier d'un État sur son produit national brut ${ }^{59}$. Les auteurs s'intéressent cependant aux sources externes de financement, aux fonds provenant d'outsiders pour reprendre les termes utilisés dans l'étude. Ils souhaitent dès lors exclure de leur mesure la part de capital détenue par les insiders. Afin d'estimer la part de capital détenue par ces insiders, LLSV analysent l'actionnariat des dix plus grandes sociétés privées cotées de chaque État et calculent la moyenne de la part détenue par les trois plus grands actionnaires de chacune de ces sociétés (ces trois plus grands actionnaires correspondent donc aux insiders) pour ensuite soustraire cette moyenne au capital de chaque société étudiée ${ }^{60}$. Les deuxième et troisième instruments de mesure sont

57. R. La Porta, F. Lopez-de-Silanes, A. Shleifer \& R. Vischny, « Legal Determinants of External Finance », op. cit., note 39, pp. 1131-1150.

58. Cf. infra point 4.1.

59. Les données datent de 1994.

60. Les auteurs sont conscients que la proportion de financement externe reste surévaluée puisqu'ils ne prennent pas en compte les participations croisées et que l'échantillon utilisé pour calculer le correctif comprend les plus grandes sociétés qui sont, comme nous l'avons vu (cf. supra point 3.1.1.), plus susceptibles de détenir un actionnariat dispersé. 
le nombre d'entreprises nationales cotées sur la population et le nombre de premiers appels publics à l'épargne pendant une année ${ }^{61}$ sur la population. Ces deux dernières variables reflètent le nombre et le flux de nouvelles entreprises qui obtiennent des financements externes (via le marché boursier donc) sous forme de capital à risque.

Mesurer le financement par crédit est plus complexe, car les informations bancaires sont difficilement accessibles. Les chercheurs se fondent donc sur le total de la dette bancaire du secteur privé et sur la valeur nominale totale des obligations dans chaque État. L'addition de ces deux valeurs divisée par le produit national brut est considérée comme un indicateur pertinent.

LLSV reprennent également des données préalablement utilisées, telles que l'origine juridique de chaque État, le critère de rule of law $w^{62}$ ainsi que l'Antidirector Rights Index et le Creditor Rights Index pour estimer le degré de protection accordé aux investisseurs des différents États, dans le but de souligner la corrélation entre le degré de protection accordé aux investisseurs (lui-même corrélé à l'origine juridique) et l'importance des marchés.

Les premiers résultats sont similaires à ceux des précédentes études. Les États de Common Law offrent à leurs entreprises le meilleur accès au financement par capitaux à risque et les États civilistes de tradition française, le pire. La pauvre protection juridique accordée aux actionnaires pourrait être un facteur explicatif. Le financement par crédit est également plus accessible dans les pays anglo-saxons que dans ceux de tradition française, mais ce sont toutefois les États de la famille germanique qui ont le niveau d'endettement le plus élevé. Selon le Creditor Rights Index, la famille de Common Law offre pourtant la meilleure protection aux créanciers. Le cas germanique amène donc à s'interroger et sème le doute concernant l'éventuel lien de causalité entre protection juridique et développement du marché. LLSV soulignent ce «mystère ${ }^{63}$ et suggèrent très brièvement une explication qui se trouverait dans la pratique comptable allemande, qui a pour habitude de comptabiliser comme dettes des informations qui ne le seraient pas dans d'autres pays ${ }^{6465}$. Ils évacuent toutefois vite la question et considèrent, de manière générale, que «legal rules influence external finance $»^{66}$.

61. Entre 1995 et 1996.

62. Cf. supra point 3.1.2.3.

63. R. La Porta, F. Lopez-de-Silanes, A. Shleifer \& R. Vishny, « Legal Determinants of External Finance », op. cit., note 39, p. 1139.

64. R.G. Rajan \& L. Zingales, « What do we Know about Capital Structure? Some Evidence from International Data », The Journal of Finance, vol. 50, n 5, 1995, pp. 1426-1427.

65. LLSV évoquent également l'hypothèse d'un modèle économique allemand axé sur le financement bancaire, sans toutefois s'y attarder (R. La Porta, F. Lopez-De-Silanes, A. Shleifer \& R. Vishny, «Legal Determinants of External Finance », op. cit., note 39, p. 1137). Cette question est pourtant cruciale, car elle souligne les différentes formes que peut revêtir le capitalisme et les différentes manières d'atteindre un objectif économique sans pour autant s'aligner sur le modèle anglo-saxon. Nous reviendrons sur ce point dans la partie critique (cf. infra point 4.3.).

66. Ibid., p. 1139. 
En isolant les 49 États étudiés (donc en les analysant indépendamment de leur origine juridique), les auteurs remarquent une forte corrélation, qui confirme leurs attentes, entre la protection des actionnaires et l'importance du marché financier. La corrélation entre le Creditor Rights Index et le niveau d'endettement est bien plus faible.

Lorsque LLSV observent uniquement les plus grandes sociétés de chaque État, les résultats montrent un visage plus ou moins similaire en termes de capitaux à risque $^{67}$, bien que l'écart entre les différents systèmes juridiques se réduise. Les différences s'estompent par contre lorsqu'il s'agit de l'accès au crédit. Toutes les grandes sociétés y ont donc accès. Cela s'expliquerait, car les grandes sociétés ont facilement accès au financement bancaire et obtiennent généralement des prêts de la part des gouvernements.

En conclusion, un haut degré de protection accordé aux investisseurs minoritaires mène non seulement à la dispersion de l'actionnariat, mais également, de manière générale, à une croissance des marchés de capitaux et donc à une augmentation des ressources financières pour les entreprises. Le tout serait influencé, en première instance, par l'origine juridique des États. L'environnement juridique anglo-saxon favorable à la protection des potentiels investisseurs incite donc à l'investissement et, en conséquence, accroît la portée des marchés ${ }^{68}$, étant entendu que, pour les tenants de la Legal Origins Theory, l'importance des marchés financiers est essentielle pour la croissance économique d'un État.

\subsection{Sa généralisation : un système globalement efficient}

En 2008, les auteurs d'origine - à l'exception de R. Vishny - se réunissent à nouveau, non plus pour réaliser une nouvelle étude empirique, mais pour dresser un état des lieux de la Legal Origins Theory ${ }^{69}$, dix ans après les premières études consacrées à la protection des investisseurs et ses conséquences.

Entre-temps, de nombreuses études ont été menées, par les mêmes auteurs et par d'autres ${ }^{70}$, dans le but de démontrer que l'influence de la Legal Origin ne se limite pas à la protection des investisseurs, mais s'étend à de nombreux autres

67. Les variables utilisées sont la capitalisation boursière des sociétés étudiées sur le total de leurs ventes et la capitalisation boursière des sociétés étudiées sur le total de leur cash-flow. Lorsque l'on applique le second ratio, la culture juridique d'ascendance germanique obtient un résultat supérieur, que les auteurs expliquent par l'évaluation très élevée des marchés japonais et taïwanais.

68. R. La Porta, F. Lopez-de-Silanes, A. Shleifer \& R. Vischny, « Legal Determinants of External Finance », op. cit., note 39, p. 1149.

69. R. La porta, F. Lopes-de-Silanes \& A. Shleifer, « The Economic Consequences of Legal Origins », Journal of Econonomic Litterature, vol. 46, $\mathrm{n}^{\circ} 2$ 2, 2008, pp. 285-332.

70. L'un des principaux contributeurs absents des premières recherches est Simeon Djankov. Chef économiste à la Banque mondiale, il fut l'un des initiateurs des rapports Doing Business (cf. infra point 3.3.). Il fut par ailleurs ministre des Finances de la République de Bulgarie. Son influence politique est donc considérable. 
domaines. L'origine juridique aurait par exemple une influence sur le caractère public ou non des banques ${ }^{71}$, sur le formalisme et les coûts nécessaires à la création de nouvelles entreprises ${ }^{72}$, sur la régulation du marché du travail ${ }^{73}$ ou encore sur la part détenue par l'État dans les médias ${ }^{74}$.

Chacune des études réalisées suit le même schéma. Dans un premier temps, les auteurs observent les effets des origines juridiques d'un État sur ses normes et institutions juridiques. Dans un second temps, ils analysent les effets économiques qu'engendrent de telles normes et régulations. Ainsi, le droit des sociétés ou des valeurs mobilières affecte les marchés financiers, les barrières à l'entrée sur le marché du travail entraînent corruption et marché au noir, l'indépendance judiciaire assure une véritable protection du droit à la propriété, les lois sur la faillite influencent les crédits privés, les lois sur le travail ont des effets sur le chômage, etc. ${ }^{75}$.

Ces différentes études peuvent être classées en trois catégories. La première, à laquelle nous nous sommes intéressés en détail, comprend les questions de droit financier et l'importance de l'origine juridique sur la protection des investisseurs. Soulignons par ailleurs que d'autres études que celles abordées dans la présente contribution ont également été menées sur le sujet ${ }^{76}$. La deuxième catégorie rassemble les différentes études abordant la question de la régulation et de toute forme d'intervention étatique ${ }^{77}$. Enfin, la troisième et dernière catégorie englobe

71. R. La Porta, F. Lopez-de-Silanes \& A. Shleifer, « Government Ownership of Banks », Journal of Finance, vol. 57, $\mathrm{n}^{\circ}$ 1, 2002, pp. 265-301.

72. S. Djankov, R. La Porta, F. Lopez-de-Silanes \& A. Shleifer, « The Regulation of Entry », Quarterly Journal of Economics, vol. 117, $\mathrm{n}^{\circ}$ 1, 2002, pp. 1-37.

73. J.C. Botero, S. Djankov, R. La Porta, F. Lopez-de-Silanes \& A. Shleifer, « The Regulation of Labor », Quarterly Journal of Economics, vol. 119, n 4, 2004, pp. 1339-82.

74. S. Duankov, C. McLiesh, T. Nenova \& A. Shleifer, «Who Owns the Media ? », Journal of Law and Economics, vol. 46, n $^{\circ}$ 2, 2003, pp. 341-381.

75. R. La Porta, F. Lopez-de-Silanes \& A. Shleifer, « The Economic Consequences of Legal Origins », op. cit., note 69, p. 292.

76. LLS se sont ainsi intéressés à l'importance du droit des valeurs mobilières (R. LA PORTA, F. LOPEZ-DESilanes \& A. Shleifer, « What Works in Securities Laws? », Journal of Finance, vol. 61, n 1, 2006, pp. 1-32), Djankov et al. proposent de remplacer l'Antidirector Rights Index par l'Anti-self-dealing Index qu'ils jugent plus pertinent (S. Duankov, R. La Porta, F. Lopez-De-Silanes \& A. Shleifer, « The Law and Economics of Self-Dealing », Journal of Financial Economics, 88, 2008, pp. 430-465). L'étude de la protection accordée aux créanciers a également été approfondie (S. DJANKOV, C. McLIESH $\&$ A. Shleifer, «Private Credit in 129 Countries », Journal of Financial Economics, vol. 84, n 2, 2007, pp. 299-329), notamment à propos de l'efficacité réelle de l'exécution forcée par l'observation des taux de recouvrement d'un créancier lors d'une faillite (S. DJAnKov, O. HaRT, C. McLiesh \& A. SHLEIFER, « Debt Enforcement Around the World », Journal of Political Economy, vol. 116, n 6, 2008, pp. 1105-1149). Le tout a pour objectif de démontrer l'importance de la Legal Origin.

77. Comme le nombre de procédures nécessaires pour créer une entreprise (S. Djankov, R. LA PoRTA, F. Lopez-DE-Silanes \& A. Shleifer, «The Regulation of Entry », op. cit., note 72, pp. 1-37), la régulation du marché du travail (J.C. Botero, S. Djankov, R. LA PorTa, F. Lopez-DE-Silanes \& A. ShlEIFER, «The Regulation of Labor », op. cit., note 73, pp. 1339-82), la part détenue par les États dans les médias (S. Duankov, C. McLiesh, T. Nenova \& A. Shleifer, « Who Owns the Media ? ", op. cit., note 74, pp. 341-381), ou encore, l'une des dernières formes d'intervention de l'État dans la vie de ses citoyens, la circonscription militaire (C. Mulligan \& A. SHLEIFER, « Conscription as Regulation », American Law and Economics Review, vol. 7, n 1, 2005, pp. 85-111). 
les questions relatives au pouvoir judiciaire et à ses effets sur la propriété privée ainsi que sur l'exécution - éventuellement forcée - des contrats ${ }^{78}$. Comparée à la tradition civiliste - française en particulier -, la Common Law offrirait une meilleure protection aux investisseurs, se caractériserait par une intervention étatique moindre ainsi que par un système judiciaire moins formalisé et plus indépendant ${ }^{79}$.

L'article de 2008 marque un véritable tournant dans la Legal Origins Theory. Celle-ci ne serait en effet plus seulement pertinente pour juger l'efficience de normes individuelles, mais également pour mesurer l'efficacité et l'impact économique de l'infrastructure dans sa globalité, du système juridique dans son ensemble ${ }^{80}$. La Legal Origins Theory se distingue désormais par son caractère englobant, presque total. Plus qu'une influence sur la protection des investisseurs, les origines juridiques d'un État conditionnent le «style of social control of economic life $»^{81}$. En effet, les sociétés doivent - selon LLS - faire face à deux grandes difficultés : le désordre et les défaillances de marché, d'une part, l'abus de pouvoir étatique, d'autre part. Ces deux problèmes sont inextricablement liés puisque chercher à résoudre les désordres ou les failles du marché entraîne nécessairement un risque accru d'abus étatique et inversement. Face à ces défis, la famille civiliste française se sentirait plus concernée par le désordre, alors que la Common Law accorderait plus d'importance aux abus de pouvoir. Cela ne signifierait nullement que le Civil Law serait dictatorial ni que la Common Law serait anarchique, mais que la balance pencherait, pour chacune des familles, d'un côté opposé. Les systèmes de Common Law offriraient plus de place aux marchés, tandis que les systèmes civilistes les restreindraient et les remplaceraient par des commandements étatiques ${ }^{82}$. Si l'on en croit M. Damaska ${ }^{83}$, la Common Law se concentrerait ainsi sur la résolution a posteriori des conflits (dispute resolving) alors que le Civil Law favoriserait l'implantation de politiques nouvelles (policy implementing), dans le but de régler les problèmes de façon anticipée ${ }^{84}$.

78. Des recherches ont été menées sur la durée d'une procédure judiciaire pour expulser des locataires mauvais payeurs (S. Djankov, R. La Porta, F. Lopez-De-Silanes \& A. Shleifer, « Courts », Quarterly Journal of Economics, vol. 118, n², 2003, pp. 453-517), sur l'efficience de l'exécution forcée des contrats (S. Duankov, O. Hart, C. McLiesh \& A. Shleifer, « Debt Enforcement Around the World », op. cit., note 76, pp. 1105-1149) ainsi que sur l'indépendance judiciaire et ses protections constitutionnelles (R. La Porta, F. Lopez-de-Silanes, C. Pop-Eleches \& A. Shleifer, « Judicial Checks and Balances », Journal of Political Economy, vol. 112, n² 2, 2004, pp. 445-470).

79. R. La Porta, F. Lopez-De Silanes \& A. Shleifer, « The Economic Consequences of Legal Origins », op. cit., note 69, p. 298.

80. J. Armour, S. Deakin, P. Leye \& M. Siems, « How do Legal Rules Evolve? Evidence from a Cross-Country Comparison of Shareholder, Creditor and Worker Protection », Brigham Young University Law Review, 2009, p. 1435.

81. R. La Porta, F. Lopez-de-Silanes \& A. Shleifer, « The Economic Consequences of Legal Origins ", op. cit., note 69, p. 286.

82. S. Djankov, R. La Porta, F. Lopez-de-Silanes \& A. Shleifer, « The New Comparative Economics », Journal of Comparative Economics, vol. 31, n 4, 2003, pp. 595-619.

83. M. Damaska, The Faces of Justice and State Authority, New Haven, Yale University Press, 1986.

84. À cet égard, R. Michaels fait remarquer que M. Damaska utilise ces expressions comme des idéaux types wébériens, c'est-à-dire en tant que catégories théoriques générales, sans prétendre couvrir toutes les caractéristiques du système observé (R. Michaels, « Comparative Law by Numbers? Legal Origins Thesis, Doing Business Reports, and the Silence of Traditional Comparative Law », op. cit., p. 782). 
LLS ne prétendent pas que la Common Law serait en tout temps mieux adaptée à l'économie et dès lors plus efficiente que le Civil Law (ce dernier serait par exemple plus efficace lorsqu'un État doit faire face à une crise majeure). En période de calme toutefois, la Common Law resterait le modèle de référence : « As long as the world economy remains free of war, major financial crises, or order extraordinary disturbances, the competitive pressures for market-supporting regulation will remain strong and we are likely to see continued liberalization. Of course, underlying this prediction is a hopeful assumption that nothing like World War II or the Great Depression will repeat itself. If it does, countries are likely to embrace civil law solutions, just as they did back then ${ }^{85}$. Ils furent toutefois plus discrets à la suite de la crise financière qui suivit.

\subsection{Son impact politique : les rapports Doing Business de la Banque mondiale}

La Legal Origins Theory a pris une tout autre dimension avec la parution des rapports Doing Business de la Banque mondiale ${ }^{86}$, en particulier leurs premières versions. Alors qu'elle était cantonnée jusqu'alors au milieu de la recherche académique, où innovation et originalité sont en principe à l'honneur, la théorie est ainsi passée dans le domaine de l'expertise, qui se fait passer pour ouvertement objective et qui cherche dans le même temps à aboutir à des résultats directs et concrets.

Ces différents rapports sont rédigés par la section Private Sector Development de l'International Finance Corporation (IFC). L'IFC est une des cinq institutions constituant le groupe de la Banque mondiale, chacune d'elles étant juridiquement et financièrement indépendante. Elle réalise sa mission d'aide au développement économique par la promotion du secteur privé, plus précisément par le biais de financements directs à des conditions analogues à celles d'opérateurs privés, ainsi qu'au moyen d'expertises fournies aux entreprises et aux gouvernements. C'est dans le cadre de cette deuxième mission qu'elle a développé l'indice Doing Business ${ }^{87}$, censé refléter la conformité d'un espace économique aux attentes du secteur privé. Cet indice de compétitivité s'inscrit dans une démarche de benchmarking, où les législations sont évaluées et dès lors comparées afin de dégager un meilleur modèle.

85. R. La Porta, F. Lopez-de-Silanes \& A. Shleifer, « The Economic Consequences of Legal Origins », op. cit., note 69, p. 327.

86. Ceux-ci font en effet expressément référence à cette théorie ; du reste, Andrei Shleifer et Simeon Djankov ont participé à leur rédaction.

87. Cet indice Doing Business est calculé sur la base de plusieurs indicateurs. Le rapport de 2004 en utilise cinq : Starting a Business, Hiring and Firing Workers, Enforcing Contract, Getting Credit et Closing a Business. Le rapport de 2005 (Removing Obstacles to Growth) en ajoute deux : Registering Property et Protecting Investors. Le rapport de 2006 (Creating Jobs) porte le nombre à dix en créant les indicateurs Dealing with Licenses, Paying Taxes et Trading across Borders. Enfin, le rapport de 2012 intègre l'accès à l'électricité (Getting Electricity) dans le calcul de l'indice. Notons également que la méthode de calcul de l'indice final a fait l'objet de modifications importantes dans le rapport de 2015 (voy. International Finance Corporation, Doing Business 2015. Going Beyond Efficiency, octobre 2014). 
Dans cette perspective, les rapports Doing Business prônent une claire diminution de la réglementation. Or il se trouve que les pays qui réglementent le plus sont, d'une part, les pays à bas revenu et, d'autre part, les pays appartenant à la culture juridique civiliste, plus particulièrement à la tradition française. L'origine juridique est considérée comme "[one of] the most important variables in explaining different levels of regulatory intervention $»^{88}$. Le degré de régulation n'est dès lors pas spécifique au régime de chaque État, mais il est façonné, lui aussi, par son héritage colonial ${ }^{89}$.

La Banque mondiale prône la voie de la convergence entre les systèmes sur la base du principe one size can fit all ${ }^{90}$. Elle considère ainsi qu'un modèle fonctionnant avec succès dans un État peut être implanté, avec le même succès, dans tout autre. Il existe naturellement des contre-exemples : une des exceptions citées est la procédure de faillite, dont la transposition d'un régime sophistiqué dans un pays en développement débouche le plus souvent sur des problèmes d'inefficacité et de corruption.

Le modèle à suivre, en grande majorité inspiré de la tradition anglo-saxonne, suppose donc une claire diminution de l'intervention étatique. Certes, « $[\mathrm{g}] \mathrm{ood}$ regulation does not mean zero regulation ${ }^{91}$; néanmoins, le niveau optimal prôné par la Banque mondiale est bien inférieur à celui en vigueur dans la grande majorité des États, particulièrement dans les pays pauvres et dans les pays appartenant à la tradition juridique française. L'État doit essentiellement se concentrer sur la protection de l'intégrité de ses citoyens et de la propriété, notamment en protégeant les droits des créanciers afin qu'ils puissent effectivement recouvrer leur créance et en assurant une application efficace du droit de propriété devant les tribunaux. L'État doit, pour l'essentiel, se limiter à ses fonctions régaliennes : il doit être un "public servant, not a public master ${ }^{92}$. Quelles sont en effet les conséquences jugées néfastes d'une réglementation trop poussée?

Tout d'abord, une réglementation excessive incite au développement de l'économie informelle. Or le développement du marché noir entraîne de nombreuses conséquences néfastes pour l'économie. Premièrement, les opérateurs actifs sur ce marché ne paient pas de taxes, ce qui réduit les recettes de l'État. De plus, les efforts entrepris pour faire respecter la loi occasionnent des coûts supplémentaires. En outre, les travailleurs ne bénéficient pas des protections légales ni des avantages

88. International Finance Corporation, Doing Business in 2004: Understanding Regulation, September 2003, p. 86.

89. Ibid., p. 84 .

90. Cette expression, expressément reprise dans le rapport de 2004, ne le sera toutefois plus dans les rapports qui suivront, en partie en réaction aux critiques du monde juridique français (B. FAUVARQUECosson et A.-J. Kerhuel, «Is Law an Economic Context? French Reactions to the Doing Business World Bank Reports and Economic Analysis of the Law », The American Journal of Comparative Law, vol. 57, n 4, 2009, p. 816).

91. International Finance Corporation, Doing Business in 2004: Understanding Regulation, op. cit., p. 15 .

92. Ibid., p. 89. 
sociaux. Les consommateurs ne sont pas non plus avantagés, car ils doivent payer une prime de risque. Enfin, la peur d'être découvert empêche les entreprises de croître et conduit à la stagnation ${ }^{93}$.

La réglementation a d'autres effets pervers, particulièrement en droit du travail où elle a tendance à se retourner contre les personnes qu'elle souhaitait protéger en première intention. En effet, alors que le droit du travail a pour principal objectif la défense de la partie faible dans la relation de travail, l'instauration de règles rigides entraîne une diminution de l'embauche, ce qui touche en premier lieu les catégories les plus faibles, c'est-à-dire principalement les jeunes et les femmes, qui n'ont pas facilement accès au marché du travail. Bien que pétrie de bonnes intentions, une forte réglementation sociale se ferait au détriment d'une partie de la population, c'est-à-dire de ceux qui n'ont pas accès au marché du travail. Concrètement, Doing Business prône une «flexibilisation » du droit du travail afin de faciliter l'embauche. Ainsi, il suggère l'instauration de contrats de travail à durée déterminée et à temps partiel, accompagnée d'une réforme parallèle des contrats à durée indéterminée afin de ne pas déboucher sur un droit du travail à deux vitesses. Les facilités doivent concerner l'embauche, mais aussi le licenciement. Un employeur prendra plus facilement la décision d'engager quelqu'un s'il n'est pas poings et pieds liés, mais s'il peut aisément mettre fin à la relation en cas de mésentente ou de difficultés économiques. Enfin, les conditions de travail doivent également être assouplies : les employeurs doivent pouvoir planifier les horaires de leurs employés en fonction des heures de pointe, les jours de congés payés devraient - comme aux États-Unis - faire l'objet d'un accord entre l'employé et l'employeur et le salaire minimum devrait être revu à la baisse, quitte à être supprimé ${ }^{94}$.

Une forte réglementation est également synonyme de lourdeurs administratives, ce qui entraîne une augmentation des délais et des coûts. Pour la création d'une entreprise par exemple, le temps qui s'écoule entre les premières démarches et la naissance légale de la société représente déjà une perte de premiers clients potentiels. Doing Business désire ouvrir au maximum l'accès à l'entrepreneuriat, en diminuant, voire en supprimant, tout obstacle à la volonté d'entreprendre. À cette fin, il faudrait notamment réduire le nombre de procédures, supprimer les autorisations notariales, bannir l'intervention des tribunaux dans le processus d'enregistrement et abolir l'exigence de capital minimum même dans les sociétés à responsabilité limitée.

93. E. Mackay et S. Rousseau, Analyse économique du droit, op. cit., pp. 170-171.

94. Cette prise de position a suscité de vives réactions. Les rapports successifs ont donc tenté d'affiner cet indicateur (l'Employing Working Indicator, abrégé EWI) dans le but de se conformer aux conventions de l'Organisation internationale du travail et d'éviter de valoriser des législations certes flexibles, mais qui ne respectent pas un seuil minimum de protection sociale (International Finance Corporation, Doing Business in 2011: Making a Difference for Entrepreneurs, 2010, p. 94). Le rapport de 2010 a finalement exclu cet indicateur du calcul de l'indice Doing Business. Il fera toujours l'objet de commentaires dans les rapports qui suivront, mais il ne sera plus intégré à nouveau dans le calcul final. 
Comme nous l'avons déjà mentionné, il existe, selon la Banque mondiale, une corrélation entre ampleur de la réglementation et appartenance à une famille juridique. Les États de tradition civiliste française réglementent le plus, alors que les États nordiques et ceux de tradition anglo-saxonne offrent « the best practices in business regulation ${ }^{95}$. Ils réglementent moins, mais protègent davantage les droits de propriété par une meilleure effectivité des contrats, par de grandes facilités offertes aux créanciers pour recouvrer leur créance, notamment lors des procédures de faillite, et, en conséquence, par une grande facilité à obtenir un crédit.

Le modèle anglo-saxon est non seulement mis à l'honneur, mais, en vertu du principe one size can fit all, comme nous l'avons mentionné à propos du principe de convergence en matière de gouvernance des sociétés ${ }^{96}$, il devrait également être le modèle à suivre : les systèmes de Civil Law devraient peu à peu se convertir aux vertus de la Common Law.

\section{LES CRITIQUES ÉMISES À L'ENCONTRE DE LA LEGAL ORIGINS THEORY}

Les travaux de la Legal Origins Theory et les rapports Doing Business n'ont pas laissé le monde juridique indifférent. Les rapports Doing Business ont le taux le plus élevé de circulation parmi les publications de la Banque mondiale et, malgré la méconnaissance de certains milieux juridiques traditionnels, l'influence de cette théorie est indéniable ${ }^{97}$. De vives réactions se sont élevées de la part du monde scientifique et politique (évidemment français ${ }^{98}$, mais pas uniquement ${ }^{99}$ ) et ont donné lieu à une littérature pléthorique - certains ont poussé la critique jusqu'à la parodie, prétendant démontrer l'importance de l'origine juridique d'un État sur ses performances footballistiques ${ }^{100}$. De cette littérature, nous allons tenter de dégager les grandes lignes et de classer - si l'on ose - les critiques en six catégories.

Premièrement, nous nous intéresserons aux critiques émises sur les méthodes employées (4.1.). Nous n'entrerons toutefois pas dans l'analyse détaillée de

95. International Finance Corporation, Doing business in 2004: Understanding Regulation, op. cit., p. 89.

96. Supra point 3.1.2.4.

97. R. Michaels, « Comparative Law by Numbers? Legal Origins Thesis, Doing Business Reports, and the Silence of Traditional Comparative Law », op. cit., p. 766.

98. Pour de plus amples développements sur les nombreuses réactions du monde civiliste français, voy. B. Fauvarque-Cosson et A.-J. Kerhuel, « Is Law an Economic Context? French Reactions to the Doing Business World Bank Reports and Economic Analysis of the Law », op. cit., pp. 815 et s.

99. Le Congrès américain a par exemple réagi à propos des rapports Doing Business, souhaitant notamment que l'indicateur Employing Workers Indicator (cf. supra note 94) ne soit plus comptabilisé pour calculer l'indice général Doing Business, ce qui finit d'ailleurs par arriver (22 U.S. Code $\S 262$ : « Reform of the "Doing Business" Report of the World Bank »).

100. M. West, «Legal Determinants of World Cup Success », Michigan Law and Economics, Research Paper No. 02-009, 2002, disponible sur https://a1papers.ssrn.com/sol3/papers. cfm?abstract_id=318940. 
chacune des informations présentées dans les rapports Doing Business ainsi que dans les études empiriques de LLSV ${ }^{101}$. Bien que ces détails puissent avoir leur importance, nous préférons concentrer l'attention sur une critique plus globale de la méthode, au lieu de nous aventurer sur le terrain de la bataille portant sur l'exactitude de chaque donnée. Une fois les méthodes critiquées, nous aborderons les critiques émanant du monde du droit comparé et la méconnaissance dont celui-ci a pu faire l'objet (4.2.). Nous nous intéresserons ensuite aux questions de convergence et interrogerons la pertinence d'une solution idéale identique pour tous les systèmes juridiques (4.3.). Dans une quatrième étape, nous questionnerons les fondements mêmes de l'analyse économique du droit néo-classique : le droit a-t-il un réel impact sur l'économie ? (4.4.). Dans une cinquième étape, nous présenterons les arguments civilistes - plus particulièrement ceux émanant de l'association Henri Capitant - louant les qualités intrinsèques du Civil Law (4.5.). Enfin, le dernier point portera sur la remise en cause de l'idée même d'efficience du droit. Le droit n'a-t-il pas une valeur propre ? Peut-il être inféodé à l'économie dans une optique exclusive de minimisation des coûts ? (4.6.).

\subsection{Une méthode faussement objective}

La première critique d'ensemble s'attarde donc sur la méthodologie employée. Celle-ci se fonde exclusivement sur l'utilisation de l'économétrie, c'est-à-dire « l'utilisation de techniques mathématiques et statistiques afin d'établir et d'interpréter l'interdépendance de plusieurs phénomènes économiques $\gg{ }^{102}$. Il s'agit donc d'une analyse fondée sur l'établissement et l'interprétation de corrélations entre deux phénomènes. Il est important de ne pas confondre cette notion avec celle de causalité. Une corrélation n'est qu'une « observation induite d'un nombre infini de cas particuliers $\rangle^{103}$, il est dès lors toujours possible de rencontrer un contreexemple, tandis que la causalité est une loi et ne souffre aucune exception ${ }^{104}$. Cette remarque incite dès lors à la prudence, car, malgré l'existence d'une corrélation entre deux phénomènes, rien ne permet de déterminer que l'un est la conséquence de l'autre, ni que cette corrélation serait due à un troisième phénomène, non mentionné dans l'étude. Les travaux inspirés de la Legal Origins Theory ont pourtant

101. Sur ce point, voy. not. H. Spamann, « The "Antidirector Rights Index" Revisited », Review of Financial Studies, vol. 23, n² 2, 2010, pp. 467-486; M. GRAFF, « Law and Finance: Common Law and Civil Countries Compared. An Empirical Critique », Economica, 75, 2008, pp. 60-83.

102. Association Henri Capitant des amis de la culture juridique française, Les droits de tradition civiliste en question. À propos des rapports Doing Business de la Banque mondiale, Paris, Société de législation comparée, 2006, p. 18.

103. Ibid., p. 20.

104. Un exemple ludique fréquemment utilisé pour illustrer cette distinction est la corrélation positive observée entre le déclin du nombre de naissances et la diminution du nombre de couples de cigognes en Allemagne de l'Ouest de 1965 à 1980 (H. SiEs, « A New Parameter for Sex Education », Nature, vol. 332, 1988, p. 495). 
démontré une certaine facilité à passer de la corrélation au lien causal ${ }^{105}$. En outre, l'attention se porte essentiellement, dans les premières études, sur le développement des marchés financiers, mais le lien entre financement par capitaux externes et succès des sociétés en question, voire croissance économique globale, est éclipsé ${ }^{106}$. D'autres études ont certes démontré la corrélation entre protection des créanciers et croissance économique, mais une protection importante serait également corrélée positivement avec un risque accru de crise financière ${ }^{107}$.

Ainsi exposée, l'analyse économétrique perd une part de son autorité. Sa traduction en langage scientifique par l'intermédiaire de fonctions mathématiques n'offre en rien un caractère irréfutable aux observations qui en sont déduites. Contrairement au sentiment propagé, elle ne produit pas d'exactitude scientifique, mais des arguments pour soutenir des présupposés. Selon le rapport Henri Capitant ${ }^{108}$, les rapports Doing Business et les travaux de LLSV en général cherchent principalement à vérifier deux propositions. La première hypothèse présuppose que l'intervention du législateur est moins efficace que celle du juge ou celle du marché, étant entendu qu'une réglementation excessive serait un frein à la croissance économique. Cet idéal de déréglementation s'inscrit dans le cadre du renouveau de la pensée libérale, mais dans une vision passablement négative du rôle de l'État. Alors que l'école des welfare economics présentait l'État comme un remède aux imperfections du marché, que l'école du public choice dépeignait l'État au service de groupes d'intérêts privés en raison de lobbyings multiples, LLSV et la Banque mondiale considèrent l'État comme le serviteur de son propre intérêt, plus exactement de celui des dépositaires de la puissance publique. De manière assez cynique, les agissements de l'État sont décrits comme étant essentiellement guidés par le clientélisme, où la corruption est à l'honneur. Une réglementation excessive, multipliant obstacles légaux et autorisations administratives, serait ainsi une porte ouverte à la pratique des pots-de-vin et une opportunité d'enrichissement indu pour les agents étatiques ${ }^{109}$. Le deuxième présupposé consiste en une affirmation de la supériorité de la Common Law sur le Civil Law, particulièrement sur la tradition juridique française. En effet, la tradition juridique française serait caractérisée par

105. C.A. Wнутоск, « Legal Origins, Functionalism, and the Future of Comparative Law », Brigham Young University Law Review, 2009, p. 1895 ; ce même auteur souligne également l'importance de ne pas se limiter à la recherche quantitative - malgré l'intérêt qu'elle peut apporter au droit comparé (sur ce point, voy. également R. Michaels, « Comparative Law by Numbers? Legal Origins Thesis, Doing Business Reports, and the Silence of Traditional Comparative Law », op. cit., p. 779) -, mais également de mener des recherches qualitatives en complément afin de renforcer les probabilités de causalité.

106. J. Ohnesorge, « Legal Origins and the Tasks of Corporate Law in Economic Development: A Preliminary Exploration », Brigham Young University Law Review, 2009, p. 1632.

107. J.F. Houston, C. Lin, P. Lin \& Y. MA, « Creditor Rights, Information Sharing and Bank Risk Taking », Journal of Financial Economics, 96, 2010, pp. 485-512.

108. Association Henri Capitant des amis de la culture juridique française, Les droits de tradition civiliste en question. A propos des rapports Doing Business de la Banque mondiale, op. cit., pp. 22 et $\mathrm{s}$.

109. Ibid., pp. 24-25. 
une forte intervention étatique. Dès lors, compte tenu du premier présupposé, elle serait moins efficiente. Ici encore, LLSV et la Banque mondiale s'appuient sur une argumentation économétrique pour vérifier le bien-fondé de cette proposition.

Ce ne sont pas les outils qui sont critiqués, mais bien leur utilisation. La méthode économétrique est neutre en elle-même ; elle a d'ailleurs déjà été employée pour défendre une thèse opposée à LLSV, selon laquelle la culture d'un pays aurait plus d'impact sur son évolution que son appartenance à une famille juridique ${ }^{110}$. Le problème se situe du point de vue de la malléabilité des outils; or il se trouve que la méthode serait « sous-tendue par la volonté d'obtenir, plus que de démontrer le résultat escompté ${ }^{111}$.

Indépendamment de toute malhonnêteté intellectuelle, le risque propre à ce genre de comparaison est en outre de voir les résultats biaisés par le cadre juridique des auteurs (ce que R. Michaels dénomme le " homeward biais ${ }^{112}$ ). Les auteurs sont susceptibles de choisir les solutions proposées par leurs propres institutions, car, par un processus d'intériorisation, celles-ci leur semblent les plus adéquates. Il est vrai que l'emploi de méthodes empiriques - indépendamment de l'exactitude des données récoltées - permet d'outrepasser ce risque (par l'utilisation de ce genre de méthodes quantitatives, la marge de manœuvre du chercheur diminue nettement une fois les résultats obtenus). Du reste, si le biais ne se trouve pas dans les réponses, il peut également se glisser dans les questions. Les questions posées le sont bien souvent en fonction du cadre juridique au sein duquel les auteurs réfléchissent. Les solutions obtenues émanent dès lors le plus souvent des systèmes juridiques dont sont issues les questions. Les performances flatteuses des ÉtatsUnis et d'autres États issus de la tradition de Common Law seraient, en définitive, la conséquence directe des questions posées ${ }^{113}$.

\subsection{Une extrapolation hasardeuse à partir du droit comparé}

Classifier les États en familles selon leurs origines historiques n'a rien de nouveau. Les plus grands chercheurs en droit comparé se sont adonnés à ce type d'exercice $^{114}$. Cependant, les différentes écoles de droit comparé n'ont pas cherché à classer les systèmes en fonction d'une supposée efficience ou efficacité économique ${ }^{115}$.

110. A.N. Licht, C. Goldschmidt \& S.H. Schwartz, « Culture, Law, and Corporate Governance », International Review of Law and Economics, $\mathrm{n}^{\circ} 25,2005$, pp. 229-255.

111. Association Henri Capitant des amis de la culture juridique française, Les droits de tradition civiliste en question. À propos des rapports Doing Business de la Banque mondiale, op cit., p. 32.

112. R. Michaels, « Comparative Law by Numbers? Legal Origins Thesis, Doing Business Reports, and the Silence of Traditional Comparative Law », op. cit., p. 786.

113. Ibid., p. 787.

114. Cf. supra point 2.2.

115. S. Deakin et K. Pistor, «Introduction », in S. Deakin et K. Pistor (eds.), Legal Origin Theory, Cheltenham, Edward Elgar Publishing, 2012, p. 10. 
En outre, ce genre de classification a principalement une portée didactique. Pour reprendre les termes de R. David, «[1]a notion de "famille de droit" ne correspond pas à une réalité biologique ; on y recourt seulement à une fin didactique ${ }^{116}$. K. Zweigert et H. Kötz - pourtant principaux inspirateurs de la Legal Origins Theory - sont eux-mêmes conscients que toute classification peut certes avoir une certaine utilité pour les débutants, mais sera nécessairement remise en cause par tout comparatiste expérimenté qui n'utilisera pas ce genre de classification ou alors avec toutes « les circonspections requises pour toute tentative de classer dans un ordre schématique un phénomène social aussi complexe qu'un système juridique en constante évolution $\gg{ }^{117}$. Ces classifications générales et didactiques dont la pertinence est remise en cause par leurs auteurs mêmes sont pourtant prises pour des « hard data ${ }^{118}$ par LLSV.

En outre, la distinction Civil Law vs Common Law n'a plus tant d'intérêt, en particulier en droit économique, car les différents systèmes juridiques issus de ces traditions tendent vers une forme de convergence. La ligne de démarcation devrait plutôt être tracée entre les droits des économies capitalistes développées et ceux des pays en voie de développement ${ }^{119}$. La différence n'en reste pas moins marquante lorsqu'il est question d'aspects procéduraux, sur lesquels Legal Origins Theory insiste beaucoup du reste ${ }^{120}$.

Le caractère immuable du concept de famille juridique est ainsi remis cause par une partie importante de la doctrine. Il est d'ailleurs plus juste d'utiliser la notion dynamique de tradition juridique plutôt que celle, statique, de famille juridique $^{121}$, désormais tenue pour désuète ${ }^{122}$. S'il existe très certainement une forme de continuité qui pourrait s'exprimer sous forme de " path dependance ${ }^{123}$, cela ne signifie pas que les systèmes se définissent par des différences statiques et permanentes ${ }^{124}$. Soulignons sur ce point le contraste entre la rigidité de la Legal Origins Theory-l'origine juridique transcenderait un État et se matérialiserait dans les

116. R. DAvid et C. Jauffret-Spinosi, Les grands systèmes de droit contemporain, 9e éd., op. cit., p. 22.

117. «All the circumspection called for by any attempt to force into a schematic order social phenomena as highly complex as living legal systems », K. ZWEIGERT \& H. KöTZ, An Introduction to Comparative Law, op. cit., p. 72.

118. R. Michaels, « Comparative Law by Numbers? Legal Origins Thesis, Doing Business Reports, and the Silence of Traditional Comparative Law », op. cit., p. 782.

119. Ibid., p. 781.

120. A.L. Glaeser et A. Shleifer, «Legal Origins », Quarterly Journal of Economics, vol. 117, $\mathrm{n}^{\circ}$ 4, 2002, pp. 1193-1229 ; R. La PORTA, F. Lopes-De-Silanes \& A. Shleifer, « The Economic Consequences of Legal Origins », op. cit., note 69, pp. 285-332.

121. H.P. GlenN, « Comparative Legal Families and Comparative Legal Traditions », in M. ReimAnN \& R. Zimmermann, The Oxford Handbook of Comparative Law, New York, Oxford University Press, 2006, pp. 422 et s.

122. B. Fauvarque-Cosson et A.-J. Kerhuel, « Is Law an Economic Context? French Reactions to the Doing Business World Bank Reports and Economic Analysis of the Law », op. cit., p. 829.

123. Nous reviendrons sur ce concept, infra point 4.3.

124. R. Michaels, « Comparative Law by Numbers? Legal Origins Thesis, Doing Business Reports, and the Silence of Traditional Comparative Law », op. cit., p. 783. 
normes adoptées - et la flexibilité des normes revendiquée par les rapports Doing Business dans leur objectif de convergence ${ }^{125}$.

Dans le même ordre d'idées, le caractère à proprement exogène du droit soulève également une question. Les institutions juridiques qui auraient été « exportées » dans les États colonisés se sont indéniablement mêlées avec des traditions juridiques domestiques ${ }^{126}$. Le droit ne s'exporte pas tel quel ${ }^{127}$, il s'imprègne du terreau dans lequel il s'intègre. La mixité des systèmes est la norme et non l'exception. De plus, le droit d'un État évolue également de manière endogène, notamment en fonction de son développement économique ${ }^{128}$.

\subsection{Un modèle universellement pertinent ?}

Les partisans d'une supériorité du modèle de Common Law semblent sous-estimer une donnée importante : l'environnement juridique, politique et culturel de chaque État. L'efficacité d'un système juridique ne peut s'évaluer qu'à l'aune de son contexte institutionnel. Telle est notamment la thèse soutenue par la théorie de la path dependence - dont la traduction littérale est « dépendance du sentier » - selon laquelle les choix politiques, économiques et juridiques s'alignent nécessairement dans le sillage historique et institutionnel d'un État ${ }^{129}$.

Par exemple, en ce qui concerne les modes de contrôle de la gouvernance et d'un souhait de convergence vers un modèle de contrôle externe, c'est-à-dire tournée vers le marché ${ }^{130}$, une série d'auteurs souligne la nécessité de la prise en compte d'un cadre institutionnel plus large pour mesurer l'efficience d'un modèle de gouvernance : «L'hypothèse privilégiée par ce courant est que, vu l'enracinement politique et culturel des institutions de gouvernance, on ne peut présumer de l'efficacité du seul système américain de type outsider parce que, loin d'être le fruit d'une évolution spontanée, celui-ci est le résultat d'un cadre juridique et de

125. LLS se défendent de ce reproche en affirmant ne pas prôner une convergence totale, mais uniquement l'amélioration de certaines règles, telles que la suppression des obstacles pour créer une entreprise par exemple (R. La Porta, F. Lopes-de-Silanes \& A. Shleifer, « The Economic Consequences of Legal Origins », op. cit., note 69, p. 325).

126. R. Michaels, « Comparative Law by Numbers? Legal Origins Thesis, Doing Business Reports, and the Silence of Traditional Comparative Law », op. cit., p. 781.

127. Ibid., p. 787.

128. B. Ahlering \& S. Deakin, « Corporate Governance, Labor Regulation and Legal Origin: A case of Institutional Complementarity? », Law and Society Review, 41, 2007, pp. 865-908.

129. La culture ne doit pas non plus devenir un « anathème » aux analyses économiques (R. MiCHAELS, «Comparative Law by Numbers? Legal Origins Thesis, Doing Business Reports, and the Silence of Traditional Comparative Law », op. cit., p. 790). D'un point de vue économique, la culture peut d'ailleurs être conceptualisée comme une contrainte pour atteindre un optimum économique sous cette forme de path dependence par exemple. Une solution étrangère peut donc être efficace, mais elle ne peut être implantée sans prendre en considération les caractéristiques culturelles de l'État importateur et exportateur.

130. Cf. supra, point 3.1.2.4. 
choix politiques qu'il serait hasardeux de hiérarchiser ${ }^{131}$. Mark Roe explique, par exemple, que le modèle américain d'actionnariat dispersé est dû en réalité à la fragmentation de ses institutions financières qui résulte d'une volonté politique opposée à la concentration du pouvoir financier entre les mains des banques et d'autres grands investisseurs de contrôle. Les choix politiques des démocraties européennes se sont davantage orientés vers les relations sociales entre dirigeants et salariés, au détriment de la souveraineté actionnariale. Cette préoccupation aurait favorisé l'émergence de blocs de contrôle destinés à maîtriser les coûts de surveillance des dirigeants ${ }^{132}$. Dès lors, renforcer les droits des actionnaires aurait des effets moins importants dans les États civilistes. Une solution efficace ne peut être transposée dans un autre État sans prendre en compte le contexte socio-économique, mais également juridique de l'État importateur. L'importation de normes anglo-saxonnes dans un État d'une autre tradition n'aurait donc pas nécessairement l'efficacité escomptée $e^{133}$.

Sous un autre angle, les hypothèses de départ de la Legal Origins Theory ont été élaborées dans un cadre de pensée (économique) anglo-américain, fondé sur un idéal de libre marché, sans que les auteurs aient pris en considération d'autres variétés de capitalisme. Attaché à un financement dépendant exclusivement des marchés financiers, ce modèle théorique ne semble pas prendre en compte les différentes formes de capitalisme, voire les différentes étapes de développement du capitalisme ${ }^{134}$.

Ainsi, dans la seconde moitié du $\mathrm{Xx}^{\mathrm{e}}$ siècle, les économies de l'Asie du Sud-Est appartenant pourtant à la tradition civiliste - ont connu une forte croissance sur le long terme. Les entreprises taïwanaises et coréennes - les célèbres chaebol coréens ${ }^{135}$ - étaient essentiellement contrôlées par de grandes familles, tandis que les japonaises pouvaient compter sur un financement bancaire important. Gage de stabilité, cette situation permet aux gestionnaires de développer des stratégies sur le long terme, à l'inverse des sociétés américaines dont le management a le regard tourné vers les cours boursiers et se préoccupe essentiellement des investisseurs volatiles. Les droits des investisseurs minoritaires étaient certes peu protégés, mais les actionnaires qui souhaitaient acquérir quelques actions n'avaient pas pour intention de consolider leurs droits. Par ailleurs, leurs spéculations avaient peu d'impact sur la direction de l'entreprise puisque celle-ci disposait d'autres

131. A. Autenne, « Analyse économique des offres publiques d'achat », op. cit., p. 356.

132. Ibid., p. 358.

133. D. Berkowitz, K. Pistor, J.-F. Richard, « Economic Development, Legality and the Transplant Effect », European Economic Review, vol. 47, n 1, 200, pp. 165-195 ; S. DEAKIN, P. SARKAR \& A. Singh, «An End to Consensus? Legal Origins Theory and the Selective Impact of Legal Reform on Financial Development », University of Cambridge Faculty of Law, Research Paper No. 40, 2011, disponible sur https://a1papers.ssrn.com/sol3/papers.cfm?abstract_id=1934737.

134. J. Ohnesorge, « Legal Origins and the Tasks of Corporate Law in Economic Development: A Preliminary Exploration », op. cit., p. 1628.

135. Groupe de sociétés contrôlé par une famille fondatrice. 
sources importantes de financement. Le succès d'entreprises telles que Sony, Samsung, Toyota, Hyundai ou encore LG témoigne de l'efficacité d'un tel mode de financement ${ }^{136}$.

Pour les pays en voie de développement, la protection des actionnaires minoritaires n'est pas nécessairement la mesure la plus efficace. Le principal défi pour ces pays est d'encourager l'entrepreneuriat et, pour ce faire, d'encourager les entrepreneurs à investir leur propre capital dans leur entreprise ${ }^{137}$. L'entrepreneuriat, pourtant moteur de croissance économique, n'est d'ailleurs pas suffisamment pris en considération par les tenants de la Legal Origins Theory (il l'est en revanche dans les rapports Doing Business). Les investissements sont évidemment nécessaires et doivent être protégés, mais sans projet il n'y a pas de croissance.

En fait, la Legal Origins Theory se concentre sur les moyens, en l'occurrence la mise sur pied d'un cadre favorable à la protection des investisseurs minoritaires, plutôt que sur le but final, c'est-à-dire la croissance économique. Or, si l'adage « la fin justifie les moyens » peut s'attirer les foudres de certains moralistes, prêcher l'inverse ne nous paraît pas raisonnable. Plusieurs chemins peuvent en effet être empruntés, certains plus adaptés que d'autres en fonction du contexte socioéconomique ainsi que juridique de l'État en question.

Attardons-nous plus en détail sur cette discussion relative aux variétés du capitalisme - Varieties of Capitalism - auxquelles LLSV ne semblent pas accorder de réelle attention ${ }^{138}$. Initialement développée par P.A. Hall et D. Soskice ${ }^{139}$, cette doctrine étudie les différences et les similarités entre les institutions des différentes économies développées. L'entreprise, par son rôle crucial dans l'économie, est au centre de l'analyse. Une entreprise entretient de nombreuses relations avec les autres acteurs économiques, tels que les clients, les fournisseurs, les entreprises concurrentes (ou avec lesquelles elle collabore), les investisseurs, le gouvernement ainsi que les travailleurs. La manière dont l'entreprise coordonne ces relations permet de souligner le contexte institutionnel dans lequel elle se situe et dès lors de comparer les différentes économies nationales. Cette doctrine intègre donc l'analyse du comportement des entreprises dans l'étude des politiques économiques dans son ensemble.

Parmi les économies capitalistes développées, une distinction est opérée entre les Liberal Market Economies (LMEs) et les Coordinated Market Economies

136. J. OhNESORge, « Legal Origins and the Tasks of Corporate Law in Economic Development: A Preliminary Exploration », op. cit., p. 1630.

137. Ibid., p. 1631.

138. LLS l'évoquent brièvement dans l'état des lieux de 2008. Ils proposent en effet aux tenants de cette doctrine de s'inspirer de la Legal Origins Theory pour pouvoir distinguer objectivement les différents types d'économies (R. La Porta, F. Lopes-De-Silanes \& A. Shleifer, « The Economic Consequences of Legal Origins ", op. cit., note 69, p. 303).

139. P.A. Hall \& D. Soskice (eds.), Varieties of Capitalism. The Institutional Foundations of Comparative Advantage, Oxford, Oxford University Press, 2001. 
$(\mathrm{CMEs})^{140141}$. Alors que les entreprises des premières coordonnent principalement leurs relations en fonction du marché, celles des secondes s'appuient principalement sur des stratégies institutionnelles hors marché (centrées non pas sur l'offre et la demande, mais sur l'influence des parties prenantes). Ici encore ${ }^{142}$, ces ensembles doivent être appréhendés comme des idéaux-types. La distinction n'est en effet pas étanche et il existe de nombreuses différences entre économies d'un même ensemble ${ }^{143}$.

La manière dont sont réglés les problèmes de coordination dépend des institutions en place dans les différentes économies étudiées. Par « institutions », les auteurs entendent un « ensemble de règles, formelles ou informelles, que les acteurs suivent généralement pour des raisons normatives, cognitives ou matérielles ${ }^{144}$. Elles ne sont donc pas exclusivement juridiques, mais peuvent par exemple correspondre à une pratique habituellement suivie. Si la structure institutionnelle d'une économie ne détermine pas complètement les stratégies des entreprises, elle les conditionne à tout le moins ${ }^{145}$.

Il existe, à l'instar des biens, une complémentarité entre les différentes institutions : certaines institutions s'accordent entre elles pour une plus grande efficacité économique. Prenons l'exemple de la République fédérale d'Allemagne, économie de type coordonnée. En termes de financement, les entreprises allemandes se tournent peu vers les marchés financiers. Elles s'intéressent donc peu à la valeur de leurs titres ${ }^{146}$ et les données comptables ne représentent donc pas des critères fiables pour mesurer la performance d'une entreprise et la valeur d'un investissement. Les investisseurs doivent donc se tourner vers d'autres indicateurs, principalement des informations internes à l'entreprise. Ce type d'informations est toutefois aisément accessible en Allemagne, grâce au tissu de relations qui se file entre entreprises d'un même secteur et grâce au nombre de participations croisées entre entreprises.

140. P.A. Hall \& D. Soskice, « An Introduction to Varieties of Capitalism », in P.A. Hall \& D. Soskice (eds.), Varieties of Capitalism. The Institutional Foundations of comparative advantage, op. cit., p. 8.

141. Parmi les États de l'OCDE, six font partie des LMEs : les États-Unis, l'Angleterre, l'Australie, le Canada, la Nouvelle-Zélande et l'Irlande; dix des CMEs : l'Allemagne, le Japon, la Suisse, les Pays-Bas, la Belgique, la Suède, la Norvège, le Danemark, la Finlande et l'Autriche ; d'autres ont une position plus ambiguë : la France, l’Italie, l'Espagne, le Portugal, la Grèce et la Turquie. Ces dernières nations ont toutefois des institutions assez semblables, de telle manière que l'on pourrait parler d'un capitalisme de type «méditerranéen », marqué par un secteur agraire important et une forte intervention étatique, mais assez récente.

142. Cf. supra note 84 .

143. P.A. Hall \& D. Soskice, «An Introduction to Varieties of Capitalism », op. cit., p. 33.

144. « $[A]$ set of rules, formal or informal, that actors generally follow, wether for normative, cognitive, or material reasons » (ibid., p. 9).

145. Ibid., p. 15.

146. Remarquons toutefois que, si la valeur des titres est trop basse, l'entreprise s'expose à un risque d'OPA hostile. De telles OPA sont toutefois encadrées par un des règles juridiques strictes. En outre, le nombre important de participations croisées est un obstacle supplémentaire à ce type de prise de contrôle. 
En revanche, les entreprises américaines se financent essentiellement par le biais des marchés financiers, constitués d'un actionnariat dispersé (qui serait donc, selon LLSV, mieux protégé). Les entreprises sont dès lors particulièrement attentives à la valeur de leurs titres et, de manière complémentaire, les investisseurs accordent beaucoup d'importance aux bilans trimestriels. En outre, le salaire des dirigeants comprend souvent une part importante de stock options et les réglementations juridiques sont tolérantes envers les $\mathrm{OPA}^{147}$, même hostiles.

Ces deux exemples démontrent, d'une part, les approches différentes d'économies différentes, mais également, d'autre part, la complémentarité qui existe entre institutions d'une même économie. Une institution, prise de manière isolée, n'est donc pas meilleure qu'une autre, elle ne l'est que dans un contexte institutionnel particulier.

Cette complémentarité mène nécessairement à une forme de convergence entre les économies similaires, alors que les différences s'accentuent entre les économies différentes. Le contexte historique des différentes économies nationales conditionne toutefois les institutions - telle est l'idée de la path dependence précédemment développée - et limite donc le phénomène de convergence dû à la complémentarité institutionnelle. Ainsi, au sein même de l'économie de type coordonnée, les entreprises du Nord de l'Europe coopèrent généralement au sein du même secteur d'activité (industry-based coordination), tandis que les japonaises ou coréennes ont plutôt tendance à coopérer au sein d'un même groupe de sociétés, mais la compétition reste forte entre entreprises d'un même secteur (group-based coordination) ${ }^{148}$.

Malgré ces différences structurelles, les Coordinated Market Economies semblent être tout aussi efficaces que les Liberal Market Economies à long terme. «Although each type of capitalism has its partisans, we are not arguing here that one is superior to another $\gg{ }^{149}$. Les principaux indicateurs du bien-être économique d'un État (national well-being) - c'est-à-dire la croissance, le PIB par habitant et le taux de chômage - démontrent d'ailleurs une certaine homogénéité entre les résultats des différentes économies ${ }^{150}$.

Certaines institutions sont toutefois mieux adaptées que d'autres pour produire certains produits ou fournir certains services ; il s'agit de la théorie des avantages comparatifs institutionnels (Comparative Institutional Advantage). Tirée de la théorie des avantages économiques ${ }^{151}$, celle-ci affirme que la différence de

147. Qui, comme nous l'avons vu (cf. point 3.1.2.4.), entraînent généralement un changement de la direction.

148. En France, par contre, les entreprises entretiendraient un lien fort avec l'État, mais faible avec d'autres entreprises.

149. P.A. Hall \& D. Soskice, «An Introduction to Varieties of Capitalism », op. cit., p. 21.

150. Les auteurs font cependant remarquer qu'il existe bien évidemment d'autres indicateurs économiques qui ont une influence sur le bien-être, comme les inégalités salariales par exemple (sur ce point, les CMEs sont moins inégalitaires que les LMEs).

151. Selon cette théorie, la production à moindre coût n'a pas pour effet d'appauvrir les États, mais au contraire de les enrichir en leur permettant de se spécialiser dans la production de certains produits qu'ils pourront échanger contre d'autres biens qu'ils ne produisent pas - ou peu. 
contextes institutionnels permettrait d'expliquer la distribution géographique de la production et des différentes activités à travers le monde.

Par exemple, les économies orientées vers les marchés, grâce à la fluidité de leur marché du travail et aux pouvoirs importants dont jouissent les dirigeants, sont mieux adaptées pour faire face aux changements radicaux. Or le secteur technologique, par exemple, se caractérise par une évolution particulièrement rapide et nécessite donc une telle capacité de réaction. Les institutions des économies marketoriented seraient ainsi plus adéquates à la production de logiciels informatiques ou au développement de biotechnologies. À l'inverse, les institutions des économies coordonnées sont plus efficaces en termes de changements incrémentaux. Marquées par une plus grande stabilité, les entreprises sont plus aptes à augmenter continuellement la qualité d'un produit et entretenir une relation de long terme avec un client. Ces économies offriraient donc un avantage pour la production de machines d'usines ou encore de moteurs, qui répondent à une telle logique incrémentale.

Autre illustration, les LMEs, par leur capacité à diminuer les coûts, seraient mieux adaptées à la production de produits élastiques au prix, alors que les CMEs offriraient des produits de meilleure qualité, grâce aux relations étroites que les entreprises entretiennent avec leurs clients et leurs fournisseurs. Dans cette optique, ce n'est désormais plus «Law matters », mais bien «Institutions matter ${ }^{152}$.

Contrairement à la Legal Origins Theory, la Comparative Institutional Advantage Theory ne prône pas une convergence vers un modèle unique. Les politiques économiques gardent le même objectif d'efficacité, mais il ne se réalisera que si les politiques sont intégrées dans le contexte institutionnel existant ${ }^{153}$. L'idée de coexistence s'oppose ici à l'idée de hiérarchie entre les modèles.

\subsection{Does law really matter?}

Si la modification des règles de droit est certes un processus aisé, ses effets risquent toutefois d'être assez faibles. L'application de nouvelles règles et l'attitude de la population envers celles-ci sont bien plus difficiles à modifier. Elles sont, à tout le moins, très difficiles à mesurer. Dès lors, lorsque LLSV proclament que «transplantation involves not just specific legal rules [...] but also legal institutions [...] human capital of the participants in the legal system, and crucially the strategy of the law for dealing with new problems ${ }^{154}{ }^{15}$, ils décrivent un tableau plutôt irréaliste d'une transposition juridique ${ }^{155}$.

152. P.A. Hall \& D. Soskice, «An Introduction to Varieties of Capitalism », op. cit., p. 37.

153. Les auteurs soulignaient à cet égard le problème du Royaume-Unis dans l'intégration européenne. Un processus de convergence ne peut en effet s'opérer entre deux économies institutionnellement différentes.

154. R. La Porta, F. Lopes-de-Silanes \& A. Shleifer, « The Economic Consequences of Legal Origins », op. cit., note 69, p. 307.

155. R. Michaels, « Comparative Law by Numbers? Legal Origins Thesis, Doing Business Reports, and the Silence of Traditional Comparative Law », op. cit., pp. 776-777. 
L'importance accordée par les tenants de la Legal Origins Theory aux règles formelles (Laws on paper) est exagérée : celles-ci doivent essentiellement être étudiées dans leur mise en application et dans leur interprétation. De là découle également une croyance irrationnelle dans l'impact des modifications juridiques sur un système économique et social ${ }^{156}$. En dépit du pouvoir des normes, une économie n'est pas si malléable. L'Allemagne du $\mathrm{XX}^{\mathrm{e}}$ siècle offre un exemple particulièrement pertinent de cette théorie : cinq régimes politiques différents se sont succédé, avec des idéologies radicalement différentes, sans que les règles principales de droit et les institutions juridiques aient été modifiées ${ }^{157}$. Les études de droit comparé ont montré que les règles juridiques formelles sont compatibles avec une variété d'idéologies et qu'une réforme juridique d'envergure ne peut se limiter à l'adoption de lois sans être accompagnée de démarches plus profondes.

\subsection{Les qualités intrinsèques du modèle de Civil Law}

L'affrontement a également pris la forme d'une défense des qualités intrinsèques du Civil Law. Tel fut notamment le cas d'un collectif d'auteurs issus de la tradition française rassemblés sous les auspices de l'Association Henri Capitant des amis de la culture juridique française qui, dans un rapport de $2006^{158}$, soulignait les atouts structurels et substantiels de cette tradition civiliste.

D'abord, le droit de tradition civiliste, par le biais de la codification, est un droit accessible. La règle contenue dans un texte de loi est bien plus accessible que celle qui découle d'une décision judiciaire. Bien qu'il soit aisé de lire et de comprendre la jurisprudence de Common Law, en extraire la règle de droit est une tâche bien plus lourde, hors de portée des non-initiés. Ce problème s'amplifie par la masse jurisprudentielle qui s'accumule au fil du temps. L'excès d'informations est devenu un problème essentiel des systèmes de Common Law, bien plus encore que celui de l'inflation législative des pays de droit écrit. Cette accessibilité n'est pas seulement matérielle, elle est également intellectuelle. L'intelligibilité de la loi jouit désormais d'une portée constitutionnelle dans le système juridique français. Cette intelligibilité était déjà souhaitée par les rédacteurs du Code civil de 1804, qui se souciaient de la clarté de la loi et de son accessibilité au plus grand nombre - clarté qui pourrait peut-être même être associée au propre de la langue française. Cette double accessibilité - matérielle et intellectuelle - met en valeur le caractère davantage démocratique du Civil Law.

156. En outre, les premières études de la Legal Origins Theory portent peu d'attention à la manière dont le droit est appliqué en pratique. L'enforcement observé dans « Law and Finance », repris en partie dans « Legal Determinants of External Finance », ne l'est que de manière superficielle, sans analyse approfondie de son application et de l'interprétation des normes par les juridictions (R. Michaels, « Comparative Law by Numbers? Legal Origins Thesis, Doing Business Reports, and the Silence of Traditional Comparative Law », op. cit., p. 776).

157. Ibid., p. 777.

158. Association Henri Capitant des amis de la culture juridique française, Les droits de tradition civiliste en question. A propos des rapports Doing Business de la Banque mondiale, op. cit. 
Ensuite, le Civil Law assure une plus grande sécurité juridique. Le droit écrit permet une connaissance a priori de la règle. Celle-ci serait dès lors plus prévisible que dans les systèmes de Common Law, où elle ne se découvre que lors de la survenance d'un litige. Les systèmes civilistes privilégieraient donc la prévention à la résolution, ce qui, dans une optique d'efficience, entraîne une diminution des coûts en raison d'une moindre nécessité de recourir aux tribunaux. C'est d'ailleurs dans la même optique qu'interviennent la fonction du notaire et son rôle en matière d'authenticité. La force probante exceptionnelle que cet officier public confère aux actes prévient de nombreux conflits et évite ainsi le recours aux tribunaux. L'Association souligne que le contentieux lié aux 4,5 millions d'actes notariés dressés chaque année s'élève à $0,004 \%$, soit un taux remarquablement faible. Ce point de vue remet dès lors en doute le bien-fondé de l'affirmation de Doing Business, qui considère l'intervention notariale comme une lourdeur administrative supplémentaire et comme un fardeau inutile.

Enfin, le dernier atout loué par l'Association Henri Capitant est la flexibilité. Cette affirmation se situe certes à contre-courant de l'idée répandue selon laquelle un droit prétorien serait plus flexible qu'un droit écrit. En effet, « il faut se garder de l'erreur courante qui consiste à inférer de l'existence d'un cadre normatif souple un contenu normatif flexible et, inversement, de celle d'un cadre normatif rigide un contenu nécessairement rigide ${ }^{159}$. Les droits de Common Law, fondés sur la règle du précédent, lient tout autant les juges, si pas plus fermement encore, que les dispositions de droit écrit. Malgré son ancrage dans les textes, un droit codifié peut évoluer. Tout d'abord, par le moyen de modifications législatives, qui permettent des réformes et des innovations que la loi du précédent ne permet pas - les systèmes de Common Law ont d'ailleurs recours au droit écrit dans ce genre de situation -, mais également par l'interprétation extensive de la jurisprudence. Ainsi le Code civil, tout en ayant gardé une structure pérenne, a pu s'adapter aux évolutions que le temps lui imposait. En dehors de toute modification des textes, le caractère abstrait et général des normes écrites offre au juge un pouvoir d'interprétation et lui permet d'adapter la norme en fonction de la situation concrète qu'il doit résoudre. Enfin, le dernier argument énoncé quant à la flexibilité est le caractère supplétif, dont le domaine ne cesse de s'étendre, de la majorité des dispositions de droit privé. La règle s'applique dès lors en cas de silence des parties ou joue le rôle de substitution en cas, par exemple, de nullité d'une clause du contrat, mais il est toujours possible d'y déroger expressément. Les opérateurs économiques ne sont dès lors pas bridés par les règles que le législateur a jugées optimales. Ces règles pallient les vides sans constituer un obstacle à l'inventivité contractuelle.

Les avantages de sécurité et de flexibilité paraissent néanmoins malaisément cumulables. La sécurité juridique s'accorde en effet difficilement avec un droit flexible et malléable. Tout est certes question d'équilibre, mais l'Association Henri

159. Ibid., p. 91. 
Capitant fait fi de cette précision. Elle défend avec autant d'ardeur et de détermination la sécurité que la flexibilité, alors que la consécration de l'une entraîne nécessairement une diminution de l'autre.

L'Association Henri Capitant défend ensuite les atouts substantiels du droit civil, en prenant l'exemple du contrat. Ne pouvant recenser toutes les institutions efficientes des systèmes de type civiliste, l'Association décide de se concentrer sur le droit des contrats, base juridique de toute opération économique. Le contrat civil joue sur un subtil équilibre entre liberté contractuelle et force obligatoire. Les parties sont libres de contracter sur tout ce qui n'est pas d'ordre public ni contraire aux bonnes mœurs, mais, une fois leur parole donnée, elles sont engagées (pacta sunt servanda). Le droit des contrats doit également composer avec des exigences d'équité et de loyauté. C'est par exemple le cas dans la phase précontractuelle, malgré le fait que les parties ne soient pas encore engagées : la jurisprudence a développé un principe de responsabilité extracontractuelle en cas de rupture abusive des négociations.

À propos de la liberté contractuelle, un tempérament est apparu à travers l'avènement du droit de la consommation. L'intention de ces normes impératives est d'éviter que le déséquilibre économique préexistant au contrat se retrouve dans les termes de la convention. En soutenant cet argument, l'Association s'éloigne des arguments classiques de l'efficience, fondés en majorité sur la déréglementation et sur un idéal de liberté individuelle, mais met en valeur la notion d'égalité. La protection de la partie faible s'impose dans un souci d'équilibre du droit civil d'inspiration française. L'Association Henri Capitant s'appuie notamment sur la célèbre phrase de Lacordaire : «Entre le fort et le faible, entre le riche et le pauvre, entre le maître et le serviteur, c'est la liberté qui opprime et la loi qui affranchit ».

Le dernier avantage avancé à l'appui des qualités du Civil Law est celui du gain de temps et d'argent qu'offre le contrat dans un tel système. La possibilité de renvoyer, explicitement ou implicitement, aux textes écrits ne contraint pas les parties à devoir anticiper les moindres détails, contrairement au contrat anglo-saxon, dont la rédaction requiert un travail fastidieux. Cette économie, l'Association rappelle ${ }^{160}$ qu'on la retrouve également au plan macro-juridique, cette fois en s'appuyant sur des chiffres, sans toutefois indiquer précisément ses sources ${ }^{161}$. Alors que le coût de l'administration de la justice (qui reprend notamment les frais exposés tant par les particuliers et les sociétés que par les pouvoirs publics en avocats, en experts, pour les tribunaux, etc.) s'élève à $2,6 \%$ du produit national brut américain, il ne s'élève qu'à $0,6 \%$ en France, $0,8 \%$ en Suisse et $0,4 \%$ en Espagne. Le nombre d'avocats

160. Elle avait déjà invoqué cet argument en soulignant la prévention des procès liée, entre autres, à la connaissance a priori de la règle et à l'institution du notaire.

161. Le rapport date de 2006, donc, malgré le manque de références, ces chiffres ne peuvent qu'être antérieurs à cette date. 
aux États-Unis est de 1 pour 300 habitants, alors qu'il est de 1 pour 1700 habitants en France. Enfin, un Américain majeur sur dix est confronté à un procès chaque année, alors qu'ils ne sont qu'un sur trois cents en France.

L'Association conclut sa réflexion sur les atouts économiques du droit civil en prenant l'exemple de l'Organisation pour l'harmonisation en Afrique du droit des affaires (OHADA) qui a ouvertement choisi de s'inspirer du modèle de Civil Law, ceci à des fins économiques. Cette organisation, soutenue par le Programme des Nations Unies pour le développement (PNUD), rencontre un véritable succès. Comble de l'ironie, son impact bénéfique est reconnu par la Banque mondiale ellemême. Le droit civil jouirait donc incontestablement d'une certaine influence à travers le monde, en raison de l'efficience de ses institutions.

\subsection{Le droit n'a pas à être inféodé à l'économie}

Après s'être ainsi livrée à un exposé des qualités intrinsèques du droit civil, le défendant positivement sur le terrain de l'efficience face au modèle de Common Law, l'Association Henri Capitant s'interroge sur l'idée même d'efficience, en se demandant si le droit doit en tout état de cause être « inféodé à l'économie » ${ }^{162}$. Sa réponse est négative : elle considère en effet que le droit a une valeur propre et critique la conséquence de l'indice Doing Business qui consiste à réduire les valeurs des différents systèmes juridiques à un dénominateur commun : la recherche du moindre coût. Le « juridiquement correct» ne doit pas faire place au « juridiquement économique ». L'Association s'oppose ainsi à une forme d'abandon du droit aux mains des économistes. L'analyse économique du droit doit être envisagée de manière réciproque de telle manière qu'il soit également permis de réfléchir sur l'analyse juridique de l'économie. Le droit ne peut se limiter à l'économisme, il devrait tendre vers le juste, en considérant les rapports humains et en veillant au maintien de la paix sociale. Pour ce faire, il doit s'intéresser à toute une série d'autres facteurs tels que les données sociales, morales, démographiques, etc. L'Association loue notamment la consécration, dans la tradition civiliste, de principes tels que l'abus de droit, la responsabilité sans faute à l'époque de la Révolution industrielle ou encore la responsabilité aggravée du vendeur à l'ère de la grande consommation : tant d'initiatives qui ne sont pas guidées par l'efficience économique prise pour elle-même, mais qui se veulent fondatrices d'une société dotée d'une communauté de valeurs. Ainsi, alors que le rapport de l'Association s'ouvrait sur une épigramme de Wilde, qui reprochait aux économistes de connaître le prix des choses et non leur valeur, il se termine par une citation de Bodin qui illustre une partie de la réponse apportée par les juristes continentaux : «Il n'est de richesse que d'hommes ».

162. Association Henri Capitant des amis de la culture juridique française, « Doing Business : le 'Da Vinci Code' du droit comparé ? », Communiqué de presse, 26 avril 2006, p. 1. 


\section{LES PRÉSUPPOSÉS PHILOSOPHIQUES DE LA LEGAL ORIGINS THEORY : LA PLACE DE L'IDÉOLOGIE DANS LA PENSÉE ÉCONOMIQUE ET JURIDIQUE OU L'IMPOSSIBLE OBJECTIVITÉ}

Les différentes démarches empiriques cherchant à démontrer de manière effective la supériorité du modèle de Common Law s'inscrivent dans un cadre de pensée plus général, qui les englobe. Nous allons désormais nous intéresser à ce substrat philosophique à partir duquel s'est développée la Legal Origins Theory et qui en a orienté la démarche : c'est lui, en effet, qui permet de comprendre l'exacte portée de ce discours. Nous étudierons deux auteurs dont la réflexion a pu avoir un réel impact sur la théorie : Friedrich August von Hayek (5.1.) et Bruno Leoni (5.2.). Le premier, représentant de l'école autrichienne, s'impose comme l'un des pères du libéralisme contemporain, notamment à travers son ouvrage Law, Legislation and Liberty, dont nous nous proposons d'analyser certains points particulièrement significatifs pour notre propos. Le second, que l'on surnomme parfois «le Hayek italien », se démarque par une pensée plus radicale encore, allant jusqu'à prôner une certaine forme d' ' anarchisme juridique ${ }^{163}$ pour répondre à son idéal de liberté individuelle.

\subsection{Friedrich August von Hayek}

La pensée de Hayek s'articule autour de la distinction entre les ordres construits, d'une part, et les ordres spontanés, d'autre part. Tandis que les premiers sont le résultat d'un dessein humain, les seconds sont le produit de rapports sociaux directs entre les individus. L'ordre spontané est un ordre qui s'engendre lui-même. Les règles qui en émanent - dénommées « règles de conduite » par Hayek - sont le produit d'interactions libres entre individus. L'ordre spontané est ainsi le résultat d'une série de forces internes, contrairement à l'ordre construit qui est l'œuvre délibérée d'une volonté particulière, extérieure aux membres qui se meuvent en son sein. Bien qu'il existe une multitude d'ordres, tant spontanés que construits ${ }^{164}$, Hayek se concentre sur un ordre spontané particulier : celui du marché ${ }^{165}$. Hayek considère en effet que le marché est à l'origine d'un ordre spontané. Cette idée n'est cependant pas nouvelle : elle remonte aux origines mêmes de l'économie politique, puisqu'elle fut déjà théorisée par Adam Smith, à travers le concept de

163. F. Lafaille, « L'anarchisme juridique de Bruno Leoni », Jus Politicum, n 5, 2011, pp. 1 et s.

164. En ce qui concerne les ordres spontanés, on peut notamment citer le langage ou la morale, qui ne sont pas inventés de toutes pièces par un esprit omniscient, mais qui sont le fruit d'une évolution dont personne n'avait projeté les résultats. En ce qui concerne les ordres construits, il existe une multitude d'organisations qui fonctionnent selon ce système, telles que les associations ou les sociétés par exemple.

165. C'est sur cet ordre que nous allons nous concentrer, de manière telle que, dans la suite des développements, nous assimilerons désormais ordre spontané et ordre du marché. 
« main invisible ». Selon cette théorie, si chaque individu agit dans une perspective purement égoïste, la somme de ces comportements aveugles débouchera, comme guidés par une main invisible, sur un optimum collectif ${ }^{166}$. Cet espace d'échanges et d'interactions libres entre individus, que Smith appelait « la grande société », Hayek le dénomme kosmos et l'assimile au domaine du droit privé.

À côté de l'ordre spontané du marché, il existe des ordres construits qui prennent la forme d'organisations. Ces organisations fonctionnent principalement selon la règle du commandement, c'est-à-dire par le biais d'instructions précises destinées à une ou plusieurs personnes particulières. Ce mécanisme permet donc de se rapprocher au maximum de l'objectif souhaité. Il n'est néanmoins pas possible de tout anticiper par l'énonciation de commandements, l'organisation doit dès lors se munir de normes générales et abstraites afin d'encadrer les actions de ses opérateurs, tout en leur laissant une certaine marge de manœuvre. Les organisations, telles que les sociétés ou les associations par exemple, sont englobées dans un ordre spontané plus vaste, à savoir l'ordre du marché. Le contraire n'est néanmoins pas envisageable : l'ordre spontané ne peut pas être englobé dans une organisation. Il existe toutefois une forme particulière d'organisation qui s'immisce pour ainsi dire naturellement dans l'ordre du marché : le gouvernement ${ }^{167}$. Sa fonction coercitive est essentielle pour l'établissement, le maintien et le fonctionnement d'un ordre spontané, car celui-ci doit être encadré par des normes générales et abstraites dont le respect doit être assuré. Le gouvernement ne peut cependant pas régir l'ordre spontané au moyen de commandements, c'est-à-dire d'instructions précises destinées à une ou plusieurs personnes particulières. Or c'est ce qui se passe dans la majorité des systèmes juridiques. Hayek déplore cette tendance - très présente à son époque, due à l'influence du communisme à l'Est et de l'État-Providence en Occident - à l'extension du modèle de l'organisation aux relations privées et commerciales. De manière plus concrète, il s'insurge contre l'ingérence étatique qui, par le biais de la législation, intervient dans le domaine du droit privé en fixant des lois dotées d'objectifs concrets au lieu de se limiter à fixer les règles du jeu.

Cette tendance serait le produit d'un inconscient collectif qui assimile l'idée même d'ordre à une volonté identifiable. En règle générale, la société n'imagine pas que l'ordre puisse s'engendrer par lui-même. Hayek dénonce le rationalisme constructiviste comme responsable de cette erreur intellectuelle. Le constructivisme est, selon lui, une idéologie politique qui considère que les choix publics doivent être guidés par une volonté de construire un certain type de société. Ce mouvement prend ses racines dans le terreau du rationalisme cartésien, dont la vision était déjà incompatible avec l'idée d'ordres spontanés. En effet, Hayek considère que les ordres spontanés atteignent un tel degré d'abstraction, un tel niveau de

166. A. Sмітн, Recherches sur la nature et les causes de la richesse des nations, Paris, Alfred Costes Éditeur, 1950.

167. La notion de gouvernement rassemble chez Hayek pouvoirs exécutif et législatif. 
complexité qu' « aucun cerveau humain ne pourrait les manipuler $»^{168}$, vision difficilement concevable dans l'optique du cogito ergo sum cartésien. Cette conception est également antinomique avec le positivisme juridique de Kelsen, qui considère que l'autorité découle du droit alors que, dans l'ordre spontané, le droit découle de l'autorité ; en d'autres termes, l'ordre ne découlerait pas du droit - qui viendrait mettre fin à un chaos -, mais lui serait en quelque sorte antérieur - les normes juridiques ne feraient, finalement, que décrire et maintenir une réalité ordonnée. Hayek considère que ce contexte intellectuel est la cause des incursions du gouvernement dans les interactions privées, car il les légitime.

Hayek s'oppose donc au règne du gouvernement sur l'ordre spontané lorsqu'il agit en édictant des lois munies d'objectifs particuliers afin de contrôler les conduites individuelles. Ces commandements sont la source d'une diminution de la liberté individuelle. Hayek estime d'ailleurs que ce système politique est voué à l'échec, car il n'est pas possible d'anticiper tous les comportements. Néanmoins, l'ordre spontané, bien qu'il soit essentiellement le produit de normes endogènes émanant de relations entre individus, doit être encadré juridiquement - d'où l'intervention nécessaire du gouvernement afin d'adopter des normes écrites générales et abstraites. Si certaines règles sont spontanément suivies par tous, soit parce qu'elles reflètent l'intérêt individuel de chacun, soit parce qu'elles font partie de la tradition culturelle, c'est-à-dire qu'elles sont ancrées dans chaque individu grâce au processus d'évolution du droit spontané, d'autres doivent parfois être imposées. C'est pour cette catégorie de normes que l'intervention du gouvernement est nécessaire. Son intervention se matérialise alors par l'intermédiaire de deux types de règles : d'une part, il peut édicter des règles d'organisation, les règles de thesis, en fixant par exemple les règles de procédure et le statut des tribunaux; d'autre part, il peut adopter des lois écrites qui sont en fait la transcription de règles de conduite, les règles du nomos, qu'il ne fait que découvrir et officialiser puisqu'elles sont le produit d'un ordre spontané. C'est notamment le cas de la propriété, que Hayek considère comme un droit naturel et non comme une construction du droit positif : le gouvernement n'invente pas la propriété, il la découvre et la fait respecter. Ce processus spontané de découverte des règles reste cependant la prérogative principale du juge, pièce maîtresse de l'ordre spontané.

Le rôle du juge est en effet de découvrir les règles de juste conduite de l'ordre spontané, c'est-à-dire les règles qui sont le produit d'interactions individuelles. En cas de conflit entre deux opérateurs, le juge est saisi afin de corriger les perturbations d'un ordre qui n'a pas été agencé. Les règles de juste conduite sont d'abord le produit d'une croissance spontanée, mais leur perfectionnement nécessite l'intervention du juge. Le juge découvre le droit, l'organise, l'améliore et va parfois même jusqu'à le rectifier. Sa principale fonction est donc de dire le droit existant, même s'il dispose également d'un pouvoir créateur en cas de lacunes. Pour revenir

168. F.A. HayeK, Droit, législation et liberté, Paris, PUF, 1980, p. 129. 
à l'exemple de la propriété, tant le gouvernement, par l'adoption de règles abstraites et générales, que le juge, par une décision qui ne s'impose qu'aux parties à la suite d'une réclamation, concourent à la découverte de ce droit, à en fixer les contours et à en assurer le respect.

Cet examen, certes succinct, de la pensée de Hayek montre l'influence du cadre de pensée dans lequel les théories de l'efficience supérieure de la Common Law ont été élaborées. La Legal Origins Theory s'est en effet développée dans un contexte de domination intellectuelle de ce type de discours, au nom de la lutte contre l'interventionnisme étatique et de la nécessité de laisser les individus s'épanouir librement dans un espace exclusivement encadré par des normes abstraites, des règles du jeu, avec un contrôle a posteriori du juge, dont la principale mission est de découvrir les normes naturelles de l'ordre spontané du marché, émanant des ajustements mutuels entre individus, et de s'y conformer dans la résolution du litige porté devant lui.

\subsection{Bruno Leoni}

Bien que Bruno Leoni ne jouisse pas d'une popularité équivalente à son homologue austro-américain, sa pensée innovatrice, voire déroutante, mérite que l'on s'y attarde également. Tant il est vrai que nul n'est prophète dans son pays, son ouvrage principal, Freedom and the Law, paru aux États-Unis en 1961, ne sera traduit dans sa langue maternelle qu'au début des années 2000 et ne bénéficiera d'une traduction française qu'en $2006^{169}$. Inconnu en Europe jusqu'à cette époque, il est cependant considéré par R. Posner comme l'un des rares pères fondateurs étrangers - c'est-à-dire non américains - du mouvement Law and Economics ${ }^{170}$. Cette ignorance continentale n'est toutefois pas étonnante, tant sa conception est en totale rupture avec sa culture d'origine et, plus généralement, avec la culture civiliste. Défenseur de ce que F. Laffaille appelle une forme d' " anarchisme juridique $\gg{ }^{171}$, Leoni se dresse contre la législation écrite. Prêt à se passer de toute intervention de l'autorité étatique, à tout le moins dans le domaine des rapports privés, il prône un droit essentiellement conventionnel, fondé sur l'échange des consentements, et jurisprudentiel, fondé sur le mécanisme de la pretes $a^{172}$.

À l'instar de Hayek, Leoni se distingue par sa réflexion multidisciplinaire, mêlant droit, économie et politique. Il déplore le rideau de fer qui se dresse entre sciences juridique, politique et économique. Il reproche en particulier aux économistes qui défendent le marché de peu s'intéresser au cadre légal lié à celui-ci,

169. B. LeONI, La liberté et le droit, Paris, Les Belles Lettres, 2006.

170. F. LAFFAILLE, « L'anarchisme juridique de Bruno Leoni », op. cit., p. 3.

171. Ibid., pp. 1 et s.

172. Que l'on peut traduire par « prétention » et qui fait donc référence à la demande présentée au juge en cas de conflit entre particuliers. 
car lui considère qu'il existe une relation étroite entre l'économie de marché et un système juridique reposant sur le juge et non sur la législation. Le marché libre doit être ancré dans un système juridique fondé sur la découverte du droit et non sur l'intervention arbitraire du législateur : «Aucun marché libre n'est vraiment compatible avec un processus d'élaboration de la loi centralisé par les autorités ${ }^{173}$.

Farouchement opposé au positivisme de Kelsen, Leoni prend comme modèles la Common Law et le droit romain, à tout le moins avant qu'ils ne soient respectivement dénaturés par l'accroissement continu du droit statutaire et par les codifications de l'empereur Justinien. En effet, s'il est si attaché à ces systèmes, c'est parce que le droit y est découvert et non décrété. Si l'empereur Justinien avait pour objectif de rassembler le droit des jurisprudents romains, tout comme les codifications du XIX ${ }^{\mathrm{e}}$ siècle avaient pour objectif de compiler les règles du passé, afin de les organiser et d'éviter toute incohérence, le simple fait de la codification, c'est-à-dire l'attachement du droit à des textes écrits, déclenche une transition du droit ouvert et spontané vers un droit clos et prévisible. Compte tenu de son évolution constante, la traduction du droit non écrit dans un système clos engendre la promulgation de nombreuses lois pour s'adapter à ces changements. Au fil du temps et de l'accumulation des normes, la loi écrite finit par devenir le seul droit pris en compte et, prise en elle-même, par prendre le pas sur le processus de découverte du droit spontané, non écrit.

Désormais, la législation franchit un pas supplémentaire, puisqu'elle ne se borne plus à organiser les lois du passé, mais devient l'expression directe de la volonté de ses auteurs. Le législateur ne se contente plus de découvrir le droit, il énonce ce qui doit être. Leoni juge cette intrusion comme un véritable obstacle à la liberté individuelle, puisqu'elle substitue au choix individuel le choix d'une personne tierce.

Le caractère représentatif des démocraties occidentales n'enlève rien au problème. La démocratie est elle-même un obstacle à la liberté individuelle, tant par son caractère indirect que par la suprématie de la loi de la majorité. Tout d'abord, en ce qui concerne le concept de représentation, d'où découle le caractère indirect des démocraties contemporaines, Leoni dénonce ce « lien mystique » qui relierait le représentant au représenté, offrant au premier la capacité d'agir dans l'intérêt du second. Il dénonce l'artifice selon lequel les élus agiraient dans l'intérêt général : «Personne n'est mieux à même de connaître sa volonté propre que soi-même ${ }^{174}$. Du reste, même si la volonté exprimée par les représentants exprimait adéquatement celle des représentés ou si la démocratie fonctionnait de manière directe, le problème majeur persisterait: la loi de la majorité. Leoni s'oppose ainsi à A. Downs, qui, partant du principe que tous les individus ont le même poids, considère que la meilleure solution est celle qui sacrifie le choix du plus petit nombre.

173. B. LeONI, La liberté et le droit, op. cit., p. 151.

174. Ibid., p. 198. 
Leoni stigmatise le système majoritaire comme l'oppression de la majorité sur les minorités. Pour prendre un exemple chiffré, dans le cadre d'une délibération, une majorité de cinquante-cinq pour cent possède un poids politique s'élevant en réalité à cent pour cent, tandis que le poids politique des quarante-cinq pour cent restants est, en définitive, nul.

Leoni suggère donc de déplacer le centre de gravité des systèmes juridiques de la législation vers l'ordre spontané, où le droit découle des ajustements mutuels et est découvert par le juge. La souveraineté doit être individuelle. Chacun doit être libre de pouvoir faire ses propres choix, sans avoir à subir la volonté d'un autre, si ce n'est éventuellement celle du juge. Néanmoins, l'institution judiciaire fonctionne selon le mécanisme de la pretesa : le juge n'intervient, de toute façon, que si l'on s'adresse à lui. En cas de requête, le rôle du juge se cantonne au rôle de spectateur. Il ne fait que découvrir le droit qui découle de l'ordre spontané, en se fondant notamment sur des décisions antérieures. Leoni nie ainsi, ou feint d'ignorer, le pouvoir en réalité discrétionnaire du juge : son rôle par rapport au droit serait le même que celui d'un linguiste par rapport au langage ou d'un statisticien par rapport à l'économie : il ne serait qu'un observateur de la réalité.

Leoni prône donc une liberté individuelle qui tend vers l'absolu, de manière telle que sa réflexion se rapproche de la pensée libertarienne, terre d'anarchie juridique. Ce degré suprême de liberté s'accompagne d'une responsabilisation des individus. En ce qui concerne les conventions entre particuliers par exemple, «le contrat léonien n'est jamais léonin ${ }^{175}$, puisque le consentement des parties est la condition nécessaire et suffisante de sa légitimité. Il s'oppose ainsi à toute protection d'une éventuelle partie faible. Un contrat est souscrit librement, jamais sous la contrainte. Peu importe la situation des parties, elles ont toujours le droit de ne pas s'engager. Il reprend notamment l'exemple du Marchand de Venise de Shakespeare, dans lequel le marchand Antonio conclut un contrat avec Shylock et l'autorise à lui prélever une livre de chair en cas de défaut de paiement. Pour Leoni, Antonio était totalement libre quant à son engagement, il doit donc en assumer les conséquences.

Au grand désarroi de l'auteur italien, les lois écrites sont de plus en plus nombreuses. L'inflation législative est tantôt justifiée par la complexité des organisations toujours plus évoluées, tantôt par les avancées techniques, voire par l'industrialisation : autant de motifs pour restreindre la liberté individuelle. Cette fièvre inflationniste pose d'ailleurs de sérieux problèmes quant à la sécurité juridique. Les lois sont en effet toujours sous la menace d'une modification législative. Le caractère figé de la loi n'est qu'illusoire puisque la décision d'une poignée d'hommes suffit pour qu'elle s'effondre. Face à un droit si malléable, il est donc difficile d'entreprendre des projets à long terme, car personne ne sait ce dont le paysage juridique sera fait demain. Sa vision de la certitude de la loi diffère dès lors de celle de la

175. F. Laffaille, « L'anarchisme juridique de Bruno Leoni », op. cit., p. 31. 
tradition civiliste, qui la déduit de la précision des termes utilisés par le législateur. Leoni conçoit la certitude de la loi « comme la possibilité offerte aux individus de faire des projets à long terme sur la base d'une série de règles adoptées spontanément par les gens, en commun, et éventuellement constatées par les juges au travers des siècles et des générations $\gg{ }^{176}$.

Il ne bannit néanmoins pas toute législation. Le législateur doit intervenir de manière négative pour proscrire certains comportements et fixer certaines limites, mais cette intervention doit rester exceptionnelle. Il inverse la présomption en considérant que « la loi est inutile sauf preuve contraire $»{ }^{177}$. La liberté individuelle est en effet compatible avec la volonté générale et les décisions de groupe, dès lors que celles-ci se bornent à appliquer le principe évangélique, déjà présent dans la philosophie confucéenne : " ne fais pas aux autres ce que tu ne veux pas que les autres te fassent ». Si nous prenons le cas d'un voleur par exemple, sa condamnation ne résulte certes pas de son choix individuel, mais, s'il avait lui-même été victime d'un vol, il serait en faveur d'une condamnation et naturellement d'une restitution de son bien. Cette interdiction est bel et bien une décision de groupe, puisque le voleur lui-même ne souhaite pas être condamné, mais, s'il avait lui-même subi un vol, il serait en faveur des mesures prises à l'encontre du larron. Dans ce cas, la volonté générale est compatible avec la liberté individuelle, puisqu'elle réprime des comportements réprouvés par tous, y compris même dans le chef des réprimés, si ceux-ci avaient été victimes de comportements similaires aux leurs.

La pensée de Leoni est contestable à bien des égards. Notre objectif ne se situe toutefois pas dans la critique, puisque nous nous bornons à détecter les présupposés philosophiques de la Legal Origins Theory. Néanmoins, une faiblesse particulièrement marquante mérite d'être soulignée. Leoni opère une distinction entre deux entités qui sont, en fait, inséparables : l'État, « hégémonique et prédateur », d'un côté, et le juge, « garant de la liberté individuelle », de l'autre. Or le droit jurisprudentiel, que l'on sache, est garanti par l'État. Leoni propose certes un mécanisme de nomination spontanée des juges, où ceux-ci seraient choisis par les individus en fonction de leurs compétences - comme s'opère le choix d'un médecin par exemple ${ }^{178}-$, mais il reste silencieux sur l'exécution des jugements, sur la force devant s'attacher aux décisions du juge. La sanction d'une inexécution contractuelle ne peut se limiter à une simple réputation négative (dans ce cas, l'intervention du juge n'aurait plus aucune utilité), mais appelle des sanctions le cas échéant forcées. Le juge, quel que soit son mode de nomination, est investi de son office par habilitation de l'État et ne tire sa propre utilité que d'être assuré que ses décisions seront exécutées, au besoin par la force.

176. B. LeONI, La liberté et le droit, op. cit., p. 157.

177. F. Laffaille, « L'anarchisme juridique de Bruno Leoni », op. cit., p. 19.

178. On ne peut s'empêcher de penser ici au mécanisme de l'arbitrage. 
De la pensée de Leoni ressortent différents présupposés philosophiques adoptés par les tenants de la supériorité de la Common Law. Tout comme chez Hayek, on retrouve cet attachement au juge, dont la fonction est d'intervenir a posteriori pour découvrir le droit naturel, lequel serait le produit d'interactions libres entre individus - ce système mettant en exergue la liberté individuelle. En revanche, il se fait plus radical que Hayek lorsqu'il traite de la législation. Il rejette l'idée même d'un cadre juridique de normes écrites, abstraites et générales. La loi écrite doit être exceptionnelle. Cette opposition à la législation est évidemment à rapprocher de l'opposition plus ou moins ouverte à la réglementation, cheval de bataille des rapports Doing Business. Une dernière remarque concerne son opposition à la loi de la majorité, qu'il désigne comme la dictature de la majorité. Celle-ci n'est pas sans rappeler les premières études de LLSV qui louaient la protection accordée aux actionnaires minoritaires dans les systèmes de Common Law, créatrice d'un marché financier plus efficient et, par conséquent, d'une meilleure croissance économique.

\section{CONCLUSION}

La démarche privilégiée par les tenants de la Legal Origins Theory, à la fois multidisciplinaire et comparatiste, est d'un grand intérêt pour les juristes. Elle ouvre une réflexion différente sur le droit, dont l'étude se cantonne généralement aux textes nationaux, qu'il s'agisse de sources purement internes ou de transpositions de normes internationales. Il s'agit en l'occurrence de prêter une réelle attention aux systèmes juridiques étrangers et de développer ainsi un point de vue plus large du droit en découvrant différentes manières de le penser. L'ouverture n'est pas uniquement géographique, elle est également disciplinaire. En s'ouvrant à l'économie, l'analyse proposée offre un nouveau prisme à travers lequel observer les normes juridiques. Elle souligne ainsi la nécessité pour les juristes de s'intéresser à d'autres méthodes - telle que la méthode empirique - et de développer une approche juridique de l'analyse économique du droit afin de ne pas abandonner cette discipline aux seuls économistes.

Si la Legal Origins Theory présente donc d'indéniables atouts intellectuels, elle n'en est pas moins critiquable sous certains aspects, notamment dans sa prétention à n'observer le droit qu'au seul prisme de l'économie et à instrumentaliser l'approche comparatiste dans une optique de convergence et de benchmarking, en vertu de laquelle les États sont comparés et classés comme des entreprises en milieu concurrentiel, en vue de détecter le système le plus efficient - en l'espèce le système anglo-saxon - et d'inciter les autres cultures juridiques à s'aligner sur ce dernier. L'économie doit être un outil d'analyse parmi d'autres, tandis que l'approche comparatiste doit tenir compte des spécificités culturelles, historiques et institutionnelles de chaque État. 
$\mathrm{Au}$ fond, c'est une certaine représentation de l'économie et du droit, c'està-dire une idéologie, qui, sous le couvert de l'objectivité statistique, est ainsi privilégiée. Les études de LLSV et, avec plus d'insistance encore, les rapports de la Banque mondiale prétendent énoncer l'objectivité en toute objectivité. Leurs auteurs affirment énoncer la vérité, non pas de manière biblique, mais sous le couvert de la science économique. Or, sans tomber dans un lyssenkisme ${ }^{179}$ de mauvais aloi, on peut avancer qu'aucune science sociale (et peut-être même aucune science de manière générale) n'est axiologiquement neutre. Les sciences sociales ont d'emblée un caractère politique, qu'il s'agisse de l'économie ou du droit, ne fût-ce que par les postulats, le plus souvent implicites, à l'origine d'une démonstration.

Cela nous conduit à une dernière réflexion, qui porte sur l'influence des présupposés philosophiques à partir desquels les théories sont le plus souvent élaborées dans le domaine des sciences sociales. Tout auteur, toute subjectivité part, la plupart du temps de façon non explicite, d'une série de présupposés, non scientifiquement démontrés, ni même démontrables. En l'occurrence, la Legal Origins Theory a été élaborée dans le cadre d'un discours de facture libérale inspiré, d'une manière ou d'une autre, par Hayek et Leoni. Si l'on modifiait le cadre et si l'on adoptait celui au sein duquel s'épanouit, par exemple, la mouvance civiliste, le résultat de l'étude eût été bien différent. D'où une double remarque de type épistémologique pour finir: d'une part, la qualité d'une recherche devrait aussi se mesurer au degré de transparence que le chercheur pratique quant aux présupposés qui guident son travail ; d'autre part, faute pour une théorie de se dévoiler de la sorte, il revient alors à ceux qui l'étudient de procéder à cette mise à nu.

\section{SUMMARY: THE LEGAL ORIGINS THEORY: LAW, ECONOMY, IDEOLOGY}

The Legal Origins Theory argues that the legal origins of a state have a significant influence on its economic growth. This theory originated mainly and prospered in the field of company law. A group of researchers has enquired about the degree of protection granted to minority shareholders in various states throughout the world. They concluded that states belonging to the legal family of common law granted the best protection, sourcing an efficient market and easing their economic growth. The Legal Origins Theory spread out widely and expanded to several other legal domains, while continuing to emphasize and highlight the efficiency of the common law. It was furthermore at the heart of the "Doing Business" reports of the World Bank. This theory has led to many critiques, which the present paper

179. En référence au biologiste Trofim Lyssenko, dont les thèses furent imposées en URSS en raison de leur caractère conforme à l'idéologie communiste. 
aims to show. Beyond this analysis and the review of criticisms, our study finally attempts to identify the philosophical assumptions that support the Theory of Legal Origins, through the review of the developments authored by Friedrich von Hayek and Bruno Leoni.

Mots-clés : Legal Origins Theory, droit comparé, analyse économique du droit, influences philosophiques

Keywords: Legal Origins Theory, Comparative Law, Law and Economics, Philosophical Influences 